\title{
EVIDENCE FOR A MILKY WAY TIDAL STREAM REACHING BEYOND 100 kpc
}

\author{
A. J. Drake ${ }^{1}$, M. Catelan ${ }^{2,3}$, S. G. Djorgovski ${ }^{1}$, G. Torrealba ${ }^{2,3}$, M. J. Graham ${ }^{1}$, A. Mahabal ${ }^{1}$, \\ J. L. Prieto ${ }^{4}$, C. DonaleK ${ }^{1}$, R. Williams ${ }^{1}$, S. Larson $^{5}$, E. Christensen ${ }^{5}$, and E. Beshore ${ }^{5}$ \\ ${ }^{1}$ California Institute of Technology, 1200 E. California Blvd., CA 91225, USA \\ ${ }^{2}$ Pontificia Universidad Católica de Chile, Departamento de Astronomía y Astrofísica, Facultad de Física, \\ Av. Vicuña Mackena 4860, 782-0436 Macul, Santiago, Chile \\ ${ }^{3}$ The Milky Way Millennium Nucleus, Av. Vicuña Mackenna 4860, 782-0436 Macul, Santiago, Chile \\ ${ }^{4}$ Department of Astronomy, Princeton University, 4 Ivy Lane, Princeton, NJ 08544, USA \\ ${ }^{5}$ Department of Planetary Sciences, The University of Arizona, Lunar and Planetary Laboratory, 1629 E. University Blvd., Tucson AZ 85721, USA \\ Received 2012 September 3; accepted 2013 January 24; published 2013 February 27
}

\begin{abstract}
We present the analysis of 1207 RR Lyrae found in photometry taken by the Catalina Survey's Mount Lemmon telescope. By combining accurate distances for these stars with measurements for $~ 14,000$ type-ab RR Lyrae from the Catalina Schmidt telescope, we reveal an extended association that reaches Galactocentric distances beyond $100 \mathrm{kpc}$ and overlaps the Sagittarius stream system. This result confirms earlier evidence for the existence of an outer halo tidal stream resulting from a disrupted stellar system. By comparing the RR Lyrae source density with that expected based on halo models, we find the detection has $\sim 8 \sigma$ significance. We investigate the distances, radial velocities, metallicities, and period-amplitude distribution of the RR Lyrae. We find that both radial velocities and distances are inconsistent with current models of the Sagittarius stream. We also find tentative evidence for a division in source metallicities for the most distant sources. Following prior analyses, we compare the locations and distances of the RR Lyrae with photometrically selected candidate horizontal branch stars and find supporting evidence that this structure spans at least $60^{\circ}$ of the sky. We investigate the prospects of an association between the stream and the unusual globular cluster NGC 2419.
\end{abstract}

Key words: galaxies: stellar content - Galaxy: formation - Galaxy: halo - Galaxy: stellar content - Galaxy: structure - stars: variables: RR Lyrae

Online-only material: color figures, machine-readable tables

\section{INTRODUCTION}

The study of the formation, composition, mass, and kinematics of galaxy halos are among the most active areas of modern astrophysical research. The formation of galaxy halos are now widely believed to be due to hierarchical structure formation (e.g., Freeman \& Bland-Hawthorn 2002) where galaxies are produced by the combination of a monolithic collapse (Eggen et al. 1962) and the accretion of galactic fragments (Searle \& Zinn 1978). For the halo of the Milky Way, all aspects of the halo can be probed by studying the nature of the remnants of disrupted dwarf galaxies.

Numerous tidal streams and dwarf galaxies have been discovered within the Galactic halo in the last 20 years (e.g., Ibata et al. 1994, 2001; Vivas et al. 2001; Grillmair 2006; Newberg et al. 2009). The most well studied of these is the Sagittarius dwarf galaxy (Sgr; Ibata et al. 1994) and its associated tidal stream. The Sgr streams have been traced on large scales using blue horizontal branch (BHB) stars (Newberg et al. 2003), M-giants (Majewski et al. 2003), and main-sequence turn-off (MSTO) stars (e.g., Belokurov et al. 2006; Koposov et al. 2012). The Sgr system has also been studied using RR Lyrae (RRL; Vivas \& Zinn 2006; Miceli et al. 2008; Sesar et al. 2010). Other halo streams and structures discovered recently include a Virgo stellar stream (VSS; Vivas \& Zinn 2006, 2008), a Virgo overdensity (VOD; Newberg et al. 2002, 2007), an overdensity in Pisces (Sesar et al. 2007; Kollmeier et al. 2009) and a Monoceros stream (Newberg et al. 2002; Majewski et al. 2003). Additionally, a Cetus stream has been discovered in the south (Newberg et al. 2009; Koposov et al. 2012) and also evidence for an overdensity dubbed the Hercules-Aquila Cloud (Belokurov et al. 2007).
Although the Sgr stream system has been the focus of much study, since the structure extends completely around the Galaxy, it has yet to be fully mapped in velocity, metallicity, and distance. Furthermore, the cause of the bifurcation in Sgr stream stars discovered by Belokurov et al. (2006) has yet to be explained. Additionally, Newberg et al. (2003) proposed the existence of a stream of stars associated with the Sgr dwarf galaxy at Galactocentric distances of $90 \mathrm{kpc}$. These authors photometrically identified candidate BHB stars in Sloan Digital Sky Survey (SDSS) data and found evidence for an overdensity of stars with $g \sim 20.5$. This overdensity was found using SDSS data covering $110^{\circ}<\alpha<130^{\circ}, 20^{\circ}<\delta<50^{\circ}$. Newberg et al. (2003) attributed this feature to a trailing arm of the Sgr stream, yet also noted a possible link to the globular cluster NGC 2419. Their analysis showed evidence for a feature visible across $\sim 20^{\circ}$ along the Sgr plane. Newberg et al. (2007) continued this work and found additional evidence for what they called the Sgr trailing tail using BHB candidates selected from SDSS Data Release 5 (DR5; Adelman-McCarthy et al. 2007). More recently, Ruhland et al. (2011) found additional evidence for this overdensity using candidate BHB stars selected from the larger SDSS DR7 data set. As with Newberg et al. (2003, 2007), these authors found a stream of BHB candidates to be located at heliocentric distances of $60-80 \mathrm{kpc}$ with $3.8 \sigma$ significance. Within their analysis the feature was seen to span $\sim 90^{\circ}$ of the sky in the region $110<\alpha<200^{\circ}$. Ruhland et al. (2011) also compared this extended stream with five sets of published numerical simulations of the Sgr stream. They found that none were able to explain the existence of Sgr stream stars in the location observed.

Current evidence for an outer Sgr stream/tidal-tail remains based purely on photometrically selected BHB stars. 
As demonstrated by Sirko et al. (2004) and Ruhland et al. (2011), it is not possible to select a $100 \%$ pure set of BHB stars using SDSS photometry alone. Depending on the color cuts used one has to accept varying levels of contamination blue straggler (BS) stars which far out number BHB stars at the greatest detection completeness. With strict color cuts, which remove a large fraction of the BHB stars, the level of BS star contamination can be reduced to $30 \%$ (Ruhland et al. 2011). However, to retain a large number of BHB stars, a $50 \%$ or greater contamination level must be accepted (Brown et al. 2005; Bell et al. 2010).

In contrast to photometric selection, it is possible to select BHB stars with much less contamination by combining color selection with high signal-to-noise $(\mathrm{S} / \mathrm{N})$ spectra because $\mathrm{BHB}$ stars have significantly lower surface gravity than the BS stars (Yanny et al. 2000). The SDSS SEGUE-I and SEGUE-II projects (Yanny et al. 2009) undertook spectroscopy of 300,000 stars with an aim of identifying BHB stars and trace Galactic halo structure. However, while radial velocities are available for stars to $g \sim 20.3$ (Yanny et al. 2009), the $\mathrm{S} / \mathrm{N}$ required to accurately measure $\log (\mathrm{g})$ limits the survey to BHB stars with $g<19.5$ $(d \sim 60 \mathrm{kpc})$. Almost all of the BHB candidates discovered by Newberg et al. $(2003,2007)$ and Ruhland et al. (2011) are beyond this limit and do not have measurements of $\log (\mathrm{g})$ to confirm their nature. Additionally, while the SDSS SEGUE surveys cover $4300 \mathrm{deg}^{2}$, the fields cover a patchwork over the Northern sky, rather than just the Sgr stream region where this feature is located.

Like BHB stars, RRL stars also exist on the horizontal branch (HB). However, unlike BHB stars, RRL exhibit a significant level of intrinsic photometric variability. Based on their characteristic variability, RRL can be cleanly separated from other stars. Type-ab RRL stars (RRab) have absolute magnitudes of $M_{V}=0.6$ with uncertainties of $6 \%$ (Catelan 2009), This makes them excellent distance indicators. However, significant numbers of repeated observations are required to provide accurate average magnitudes.

To date, a few tens of thousands of RRL are known in dense regions near the Galactic bulge and in the Magellanic Clouds due to microlensing surveys (Soszyński et al. 2009; Pietrukowicz et al. 2012). Recently we discovered $\sim 10,000$ RRab over a large fraction of the sky (Drake et al. 2013, DR13). However, even with the DR13 RRab's most of the Galactic halo remains unexplored at heliocentric distances $>60 \mathrm{kpc}$.

In this paper we outline our search, discovery, and calibration of RRL at distances up to $100 \mathrm{kpc}$ and beyond. We will compare our discoveries with results from past surveys and undertake an analysis of the distant HB stars uncovered.

\section{OBSERVATIONAL DATA}

The Catalina Sky Survey began in 2004 and uses three telescopes to cover the sky between declination $-75^{\circ}$ and $+65^{\circ}$ in order to discover Near-Earth Objects (NEOs) and Potential Hazardous Asteroids (Larson et al. 2003). The three telescopes are each considered sub-surveys. These consist of the Catalina Schmidt Survey (CSS), the Mount Lemmon Survey (MLS), and the Siding Spring Survey (SSS). In addition to avoiding high declinations, the Galactic plane region is avoided by between 10 and 15 deg due to reduced source recovery in crowded stellar regions. All of the survey images are taken in sequences of four observations separated by 10 minutes, and all the observations are unfiltered to maximize throughput. Photometry is carried out using the aperture photometry program SExtractor (Bertin $\&$ Arnouts 1996). All the Catalina data are also analyzed for transient sources by the Catalina Real-time Transient Survey (CRTS ${ }^{6}$; Drake et al. 2009; Djorgovski et al. 2011).

In Paper I (DR13), we concentrated on analyzing RRab data from the CSS sub-survey. In this paper we mainly work with data observed by the MLS $1.5 \mathrm{~m}$ telescope. These data predominately cover ecliptic latitudes $-10^{\circ}<\beta<10^{\circ}$. For this telescope each image from the $4 \mathrm{k} \times 4 \mathrm{k}$ Catalina CCD camera covers $1.1 \mathrm{deg}^{2}$ on the sky. Observations are taken 21 nights per lunation avoiding bright time. Typical exposures of 30 s to reach $V \sim 21.5$. In total, the MLS source catalog consists of 155 million sources.

\subsection{Calibration}

As noted in DR13, the MLS data have the same photometric sensitivity as the CSS data since all observations are taken unfiltered with the same type of CCD camera. All images are processed with the same software. From DR13, the color transformation from Catalina photometric system $V_{\mathrm{CSS}}$ magnitudes to Johnson $V$ is given by

$$
V=V_{\mathrm{CSS}}+0.31 \times(B-V)^{2}+0.04 \text {. }
$$

The average $B-V$ color of RRL is about $0.3 \mathrm{mag}$ with stars varying between about 0.1 and 0.5 as they pulsate (e.g., Clube et al. 1969; Stepien 1972; Cacciari et al. 1987; Layden 1997; Nemec 2004). As the MLS data have no color information, we adopt the average values in our analysis. This gives rise to a maximum uncertainty of $\sim 0.07 \mathrm{mag}$ in the $V$ magnitudes. Combining this with the photometric uncertainty, we expect a color-based dispersion of $\sigma=0.09 \mathrm{mag}$ in our RRL photometry.

\subsection{RR Lyrae Selection}

To find RRL candidates we follow the analysis undertaken in DR13. That is, we first selected variable sources using a Welch-Stetson variability index $I_{\text {WS }}$ (Welch \& Stetson 1993). A total of 3.1 million MLS sources were selected as variable candidates using $I_{\mathrm{WS}}>0.6$. All sources were processed for periodicity using the Lomb-Scargle (LS; Lomb 1976; Scargle 1982) periodograms. In total 170 thousand sources were found to exhibit significant periodicity with a false alarm probability $p_{0}<1 \times 10^{-5}$. However, the bulk of these detections are due to the systematic sampling of the data and occur at integer day frequencies.

Here, as with DR13, we are primarily interested in RRab's since they have well-known magnitudes and characteristic light curves that are not easily mistaken for other periodic variables. After excluding the period aliases near 0.5 and 1 day, 3087 of the objects were found to have periods between 0.34 and 1.4 days. This period region was deliberately chosen to be broader than the RRab range in order to include sources found by the LS technique at aliases of their true periods.

As with the CSS sources of DR13, each of the RRab candidates was run through the AFD software (G. Torrealba et al. 2013, in preparation) to select the best period from among the best 15 given by the LS and AoV software (SchwarzenbergCzerny 1989). Briefly, this process involves Fourier fitting and iteratively rejecting bad data to determine the best period based on reduced $\chi^{2}$ values. Non-RRab sources were rejected using the M-test statistic (Kinemuchi et al. 2006, Equation (8)), as well as the Fourier fit order. This initial selection resulted in the detection of 1125 RRab candidates.

\footnotetext{
6 http://crts.caltech.edu/
} 


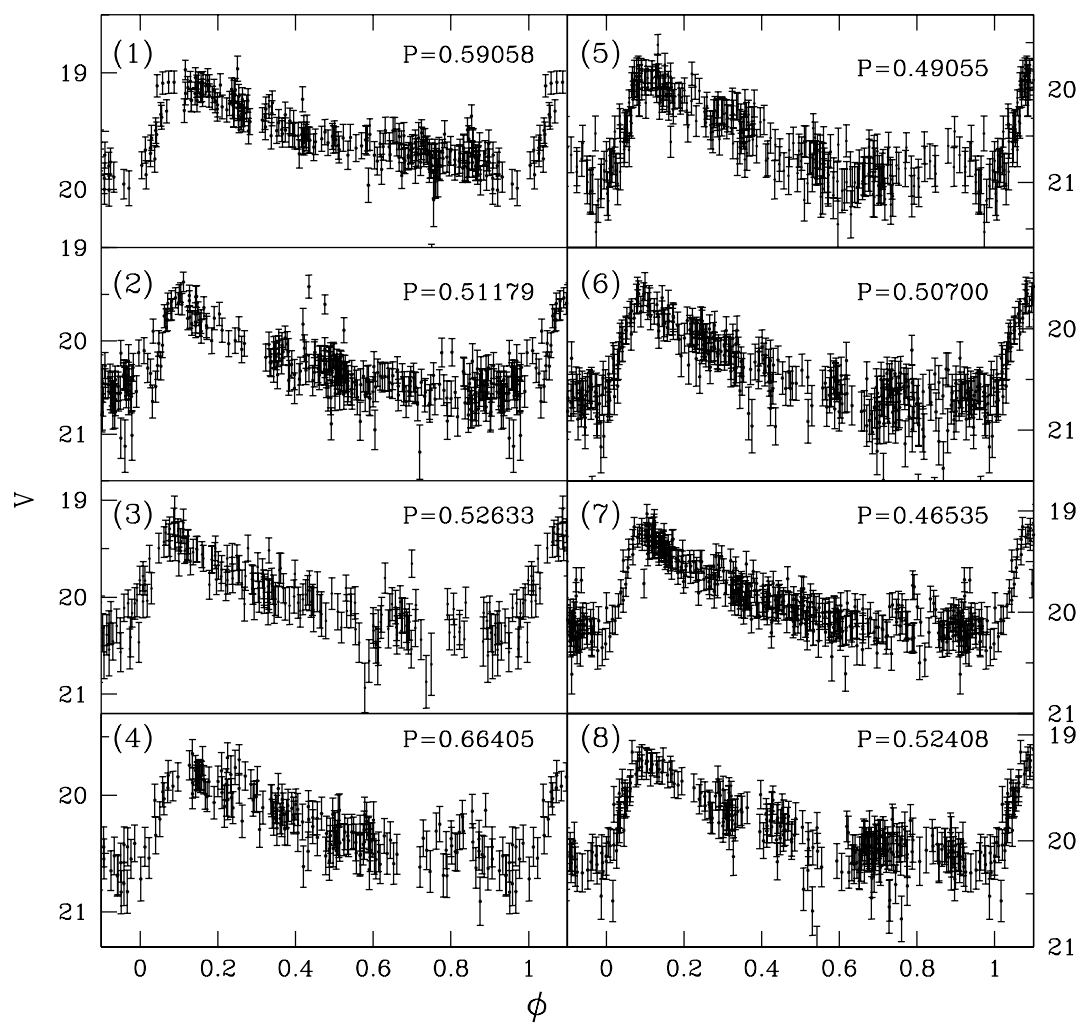

Figure 1. Period-folded light curves of eight of the Galactic halo RRab discovered in MLS photometry. Here the numbered panels are correspondingly: (1) MLS J093311.1+131726; (2) MLS J092028.4+153244; (3) MLS J050243.0+203816; (4) MLS J073511.7+185253; (5) MLS J080834.1+192509; (6) MLS J081338.1+191449; (7) MLS J074044.7+204658; and (8) MLS J043725.2+211020.

\subsection{Faint RRab Stars}

Upon reviewing the light curves of the RRab candidates we discovered a number of faint RRab's in the MLS data with $V \sim 20.5$. Such RRL were found to be mainly concentrated in the constellation Gemini. In Figure 1, we plot the phased light curves of eight of these faint stars. These distant sources are very important for defining distant streams within the Galactic halo. From simulations based on adding artificial RRab light curves to our data, we discovered that our detection sensitivity was $<50 \%$ for RRab's with $V>20$.

To improve our completeness we investigated the light curves of MLS sources with SDSS spectroscopic types A0 and F5 that had $19<V<21$. In addition, we used SDSS photometry to select stars with the same color range as the RRab stars that we had discovered. These stars were matched with MLS sources at lower variability and periodicity thresholds $\left(I_{\mathrm{Ws}}>0.3\right.$ and $\left.p_{0}<0.01\right)$ to select RRab candidates. To better constrain the variability, we combined the MLS photometry with shallower CSS data. Likewise, we searched the CSS data for additional faint RRab candidates by selecting objects with RRab colors in SDSS photometry. Furthermore, since our analysis in DR13 showed that $\sim 17 \%$ of RRab's were missed because the selection was based on LS periods, we reprocessed the CSS and MLS photometry using the AoV period-finding software.

We inspected the light curve of each RRL candidate to assess variability and then carried out period finding for all the new variable candidates. In total, approximately 6000 additional sources were searched. Each of the new sources exhibiting some sign of periodicity were searched for improved periods using our Fourier-fitting AFD process. In a number of cases the RRL were found to exhibit Blazhko phase and amplitude variations (Blazhko 1907).
In total the additional searches yielded an extra 219 MLS RRL and 2051 CSS RRab's. Of these, only 425 were previously known. As distant RRL are important for defining Halo streams we retain the 17 clear c-type RRL (RRc) that were discovered in MLS data beyond $70 \mathrm{kpc}$. A number of these have SDSS spectra. After removing non-RRL sources the final set of MLS RRL consists of 1207 stars and is presented in Table 1. The new RRab's found in CSS data are given in Table 2.

In Figure 2, we present the magnitude distribution of the MLS and the CSS RRL. The MLS histogram shows clear bumps near $V=19.5$ and $V=20.5$. The first bump is easy to understand as it is clearly due to the Sagittarius leading and trailing arms (as seen in DR13). The second peak is due to the more distant RRL that are concentrated near the Galactic anti-center. We hereafter call this the Gemini stream. In Section 5, we will investigate the origin of this feature.

\section{RRab DISTANCES}

The absolute magnitudes of RRab's are given by Catelan \& Cortés (2008):

$$
M_{V}=0.23 \times[\mathrm{Fe} / \mathrm{H}]_{\mathrm{ZW} 84}+0.948,
$$

where $[\mathrm{Fe} / \mathrm{H}]_{\mathrm{ZW} 84}$ is the metallicity in the Zinn \& West (1984) scale. The average metallicity for RRab's with SDSS spectra found by DR13 was $[\mathrm{Fe} / \mathrm{H}]=-1.55$. The comparison given in DR13 between distant halo RRab's $\left(d_{G}>33.5 \mathrm{kpc}\right)$ and the full set of RRab's showed little difference in the distribution or average metallicity. However, CSS RRab's with SDSS metallicities are stars with $V>16$, corresponding to a distance of $>12 \mathrm{kpc}$. Brighter foreground field RRab's are likely to have higher metallicities. The MLS RRab's should have a metallicity distribution similar to the larger sample of 
Table 1

RRab from MLS

\begin{tabular}{|c|c|c|c|c|c|c|c|c|c|}
\hline ID & $\begin{array}{c}\text { R.A. } \\
\left({ }^{\circ}\right)\end{array}$ & $\begin{array}{c}\text { Decl. }(\mathrm{J} 2000) \\
\left({ }^{\circ}\right)\end{array}$ & $\left(V_{0}\right)_{S}$ & $\begin{array}{c}P \\
\text { (days) }\end{array}$ & $\begin{array}{c}A \\
(\mathrm{mag})\end{array}$ & $N$ & $\begin{array}{c}d_{h} \\
(\mathrm{kpc})\end{array}$ & $A_{V}$ & $\mathrm{ID}_{\text {alt }}$ \\
\hline MLS_J000452.4+013646 & 1.21861 & 1.61300 & 18.27 & 0.540075 & 1.06 & 124 & 33.22 & 0.060 & \\
\hline MLS_J000604.5+031006 & 1.51876 & 3.16854 & 18.15 & 0.586872 & 0.95 & 81 & 31.75 & 0.038 & MG1 2425 \\
\hline MLS_J001018.0+011013 & 2.57540 & 1.17054 & 15.30 & 0.738322 & 0.45 & 144 & 8.55 & 0.046 & [SIG2009] 98874 \\
\hline MLS_J001031.0+010132 & 2.62956 & 1.02568 & 18.89 & 0.509527 & 0.87 & 140 & 44.43 & 0.046 & SDSS J001031.09+010132.4 \\
\hline MLS_J001121.0+001014 & 2.83772 & 0.17062 & 16.70 & 0.562567 & 0.88 & 140 & 16.09 & 0.063 & Loneos-RR 728 \\
\hline MLS_J001649.1+025907 & 4.20471 & 2.98534 & 16.78 & 0.635702 & 0.49 & 116 & 16.83 & 0.053 & \\
\hline MLS_J001828.2+042407 & 4.61780 & 4.40213 & 15.94 & 0.641855 & 0.40 & 93 & 11.45 & 0.045 & CSS J001828.2+042408 \\
\hline MLS_J001847.7+033347 & 4.69878 & 3.56312 & 15.75 & 0.555048 & 1.07 & 111 & 10.49 & 0.044 & CSS J001847.6+033347 \\
\hline MLS_J002035.1+011431 & 5.14626 & 1.24210 & 15.11 & 0.465817 & 1.31 & 176 & 7.89 & 0.042 & Loneos-RR 729 \\
\hline MLS_J002150.1+020042 & 5.45912 & 2.01182 & 17.36 & 0.624099 & 0.53 & 137 & 21.86 & 0.059 & Loneos-RR 739 \\
\hline MLS_J002449.8+011256 & 6.20764 & 1.21571 & 15.68 & 0.680415 & 1.00 & 137 & 10.13 & 0.049 & Loneos-RR 193 \\
\hline
\end{tabular}

Notes. Column 1 gives the MLS ID. Columns 2 and 3 give the right ascension and declination. Column 4 gives average magnitude from the Fourier fit to the light curve. Column 5 gives the period of the RRab. Column 6 gives the Fourier fit amplitude to the observed variation. Column 7 gives the number of photometric observations. Column 8 gives the heliocentric distance to the RRab. Column 9 gives the extinction based on the Schlegel et al. (1998) reddening map. Column 10 gives the IDs for sources that were previously known.

(This table is available in its entirety in a machine-readable form in the online journal. A portion is shown here for guidance regarding its form and content.)

Table 2

RRab from CSS

\begin{tabular}{|c|c|c|c|c|c|c|c|c|c|}
\hline ID & $\begin{array}{l}\text { R.A. } \\
\left({ }^{\circ}\right)\end{array}$ & $\begin{array}{c}\text { Decl. (J2000) } \\
\left({ }^{\circ}\right)\end{array}$ & $\left(V_{0}\right)_{S}$ & $\begin{array}{c}P \\
\text { (days) }\end{array}$ & $\begin{array}{c}A \\
(\mathrm{mag})\end{array}$ & $N$ & $\begin{array}{c}d_{h} \\
(\mathrm{kpc})\end{array}$ & $A_{V}$ & $\mathrm{ID}_{\text {alt }}$ \\
\hline CSS_J000343.1-134943 & 0.92972 & -13.82868 & 17.66 & 0.586691 & 0.77 & 166 & 24.98 & 0.071 & $\ldots$ \\
\hline CSS_J000357.3+171314 & 0.98886 & 17.22066 & 16.82 & 0.653923 & 0.68 & 212 & 17.09 & 0.058 & $\ldots^{\mathrm{a}}$ \\
\hline CSS_J000427.0+282435 & 1.11252 & 28.40975 & 16.23 & 0.667690 & 0.73 & 148 & 12.76 & 0.102 & $\ldots$ \\
\hline CSS_J000614.5+194136 & 1.56060 & 19.69337 & 14.18 & 0.776959 & 0.38 & 189 & 5.03 & 0.069 & $\ldots$ \\
\hline CSS_J000622.1-161859 & 1.59247 & -16.31656 & 17.48 & 0.586580 & 0.74 & 124 & 23.24 & 0.046 & $\ldots{ }^{\mathrm{a}}$ \\
\hline CSS_J000736.5-081444 & 1.90229 & -8.24558 & 16.96 & 0.594456 & 0.88 & 179 & 18.20 & 0.060 & $\ldots$ \\
\hline CSS_J000753.6-082406 & 1.97343 & -8.40185 & 18.81 & 0.666633 & 0.88 & 175 & 42.58 & 0.062 & $\ldots$ \\
\hline CSS_J000802.0-212139 & 2.00848 & -21.36106 & 15.62 & 0.633402 & 0.90 & 80 & 9.93 & 0.036 & $\ldots$ \\
\hline CSS_J000817.4+415259 & 2.07257 & 41.88319 & 16.10 & 0.645170 & 0.32 & 159 & 11.81 & 0.133 & $\ldots$ \\
\hline CSS_J001022.3-194420 & 2.59304 & -19.73891 & 16.23 & 0.676047 & 0.40 & 94 & 13.10 & 0.039 & $\ldots$ \\
\hline CSS_J001132.6+305140 & 2.88606 & 30.86132 & 16.13 & 0.619320 & 0.76 & 148 & 12.15 & 0.109 & NQ And \\
\hline CSS_J001205.3-184653 & 3.02246 & -18.78156 & 16.92 & 0.513594 & 1.13 & 111 & 17.96 & 0.050 & $\ldots$ \\
\hline CSS_J001824.6-191411 & 4.60261 & -19.23640 & 15.54 & 0.683731 & 0.62 & 111 & 9.54 & 0.046 & $\ldots$ \\
\hline CSS_J001957.6-025853 & 4.99018 & -2.98164 & 17.36 & 0.609065 & 0.47 & 155 & 21.93 & 0.059 & $\ldots^{\mathrm{a}}$ \\
\hline CSS_J002002.7+103504 & 5.01164 & 10.58448 & 17.36 & 0.647210 & 0.47 & 203 & 19.99 & 0.255 & $\ldots$ \\
\hline
\end{tabular}

Notes. Column 1 gives the MLS ID. Columns 2 and 3 give the right ascension and declination. Column 4 gives average magnitude from the Fourier fit to the light curve. Column 5 gives the period of the RRab. Column 6 gives the Fourier fit amplitude to the observed variation. Column 7 gives the number of photometric observations. Column 8 gives the heliocentric distance to the RRab. Column 9 gives the extinction based on the Schlegel et al. (1998) reddening map. Column 10 gives the IDs for sources that were previously known.

${ }^{a}$ Uncertain period.

${ }^{\mathrm{b}}$ Candidate Blazhko RR Lyrae.

(This table is available in its entirety in a machine-readable form in the online journal. A portion is shown here for guidance regarding its form and content.)

CSS sources discovered in DR13. Therefore, we adopt the average CSS metallicity and assume RRab's have magnitude $M_{V}=0.59$. This value is close to the value of 0.6 that is often adopted (e.g., Keller et al. 2008; Sesar et al. 2010). The RRL $V$ magnitudes were corrected for extinction using Schlegel et al. (1998) reddening maps. The dispersion in the metallicity from DR13 is 0.3 dex, which corresponds to a variation of 0.07 mag. The distances to individual sources are determined using

$$
d=10^{\left(\left(V_{0}\right)_{s}-M_{V}+5\right) / 5} .
$$

Here we correct the average RRab $V_{0}$ magnitudes to static values $\left(V_{0}\right)_{\mathrm{s}}$ using values derived from a polynomial fit to the amplitude corrections given by Bono et al. (1995). Combining the uncertainties from the photometric calibration and color variation of 0.09 , with the variation in metallicity we derive an overall uncertainty of $0.11 \mathrm{mag}$, corresponding to a $\sim 5 \%$ uncertainty in distance. As demonstrated by DR13, faint RRL have larger uncertainties in their average magnitude. However, these uncertainties should generally not exceed $0.2 \mathrm{mag}(\sim 10 \%$ in distance). The faintest MLS RRab in our data set have 


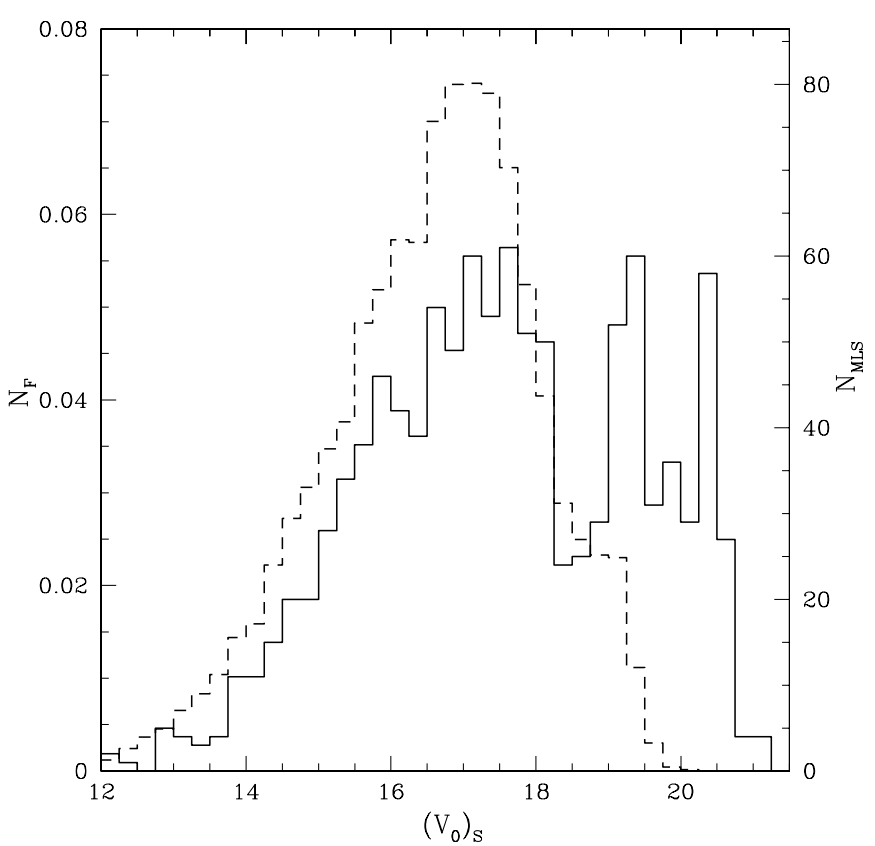

Figure 2. Distribution of RRL magnitudes. The fractional number $\left(N_{F}\right)$ of RRL static magnitudes for the 1207 MLS RRLs found in the survey are plotted with the solid line. The $\sim 14500$ CSS RRab's are plotted with the dashed line. The peak in the MLS distribution near $V=19.5$ is mainly due to RRL in the two separate arms of the Sgr stream system. The peak near mag $V=20.5$ is mainly due to the distant Gemini feature. The actual numbers of MLS RRL are noted by $N_{\text {MLS. }}$ The actual number of CSS RRab's are $N_{\text {CSS }} \sim 13.4 \times N_{\text {MLS }}$.

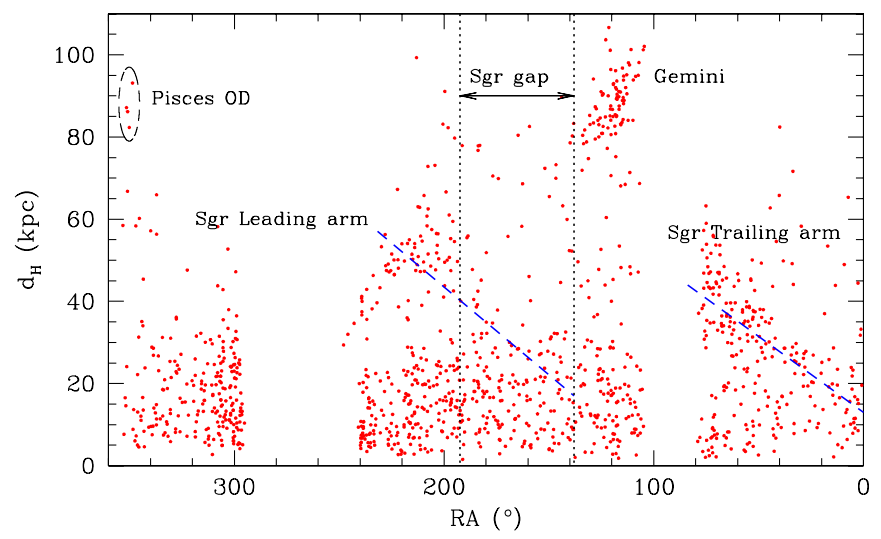

Figure 3. Heliocentric distance distribution for all the MLS RRab stars presented in this work. Each of the distances is derived from $V$ magnitudes corrected to static star values. Known features including the Sagittarius streams and Pisces overdensity (Pisces OD; Sesar et al. 2007) are marked. We also marked the central region of the Sgr streams that is not covered by the MLS data (Sgr gap). (A color version of this figure is available in the online journal.)

$\left(V_{0}\right)_{\mathrm{S}} \sim 21$ corresponding to heliocentric distances of $120 \mathrm{kpc}$. In Figure 3, we plot the distribution of the distances of MLS RRL and mark the locations of known halo features as well as the Gemini stream.

\section{COMPARISON WITH KNOWN RRab}

We matched the MLS RRab's with past RRL discoveries using the SIMBAD and the International Variable Star Index (VSX; Watson et al. 2006) databases as well as those presented by DR13. In total, there were 605 matches with past surveys of which 316 came from CSS data (DR13) and 289 were discovered in other surveys. As there is overlap between CSS and the other previously known RRab's, there was CSS photometry for 540

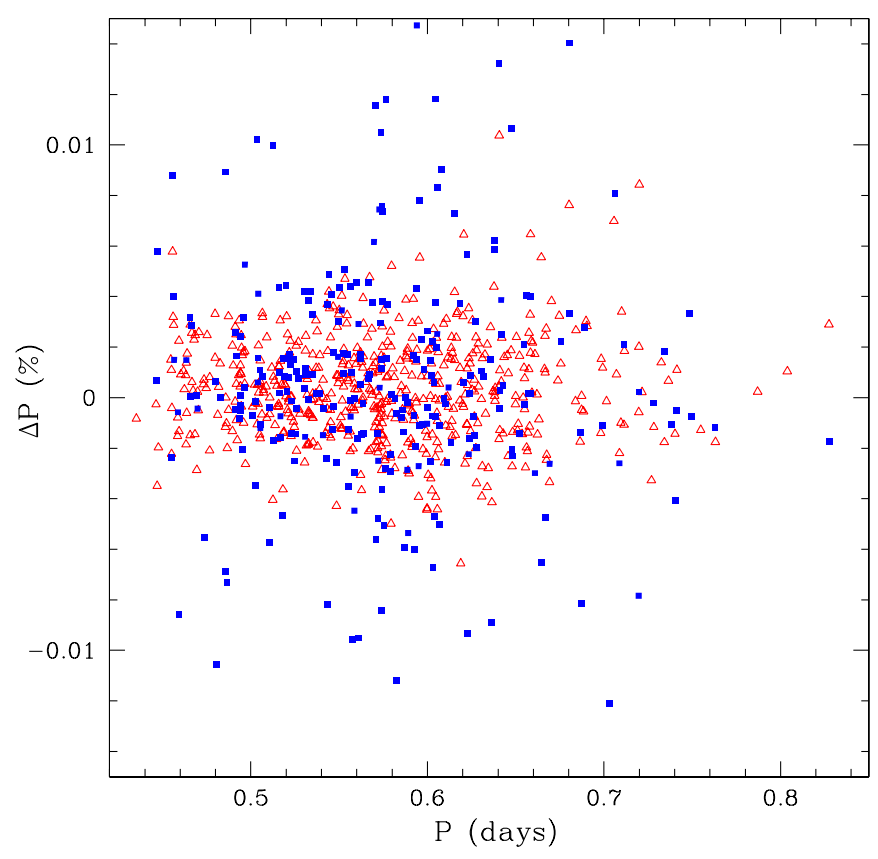

Figure 4. Comparison of MLS RRab periods. Here we plot the difference in period between that derived from MLS data and elsewhere for previously known RRab's. Periods based on the Drake et al. (2013) CSS RRab's catalog are given with triangles, while all other sources are presented as filled boxes.

(A color version of this figure is available in the online journal.)

of the MLS RRab's. Of the 289 SIMBAD and VSX RRab's, 278 of the RRab have known periods given by VSX. In Figure 4, we compare the periods derived from MLS data with previous determinations. The figure shows that the agreement between the CSS and MLS periods is much better than with other sources. Assuming the differences in periods are normally distributed, the MLS-CSS matches have $\sigma=0.0020 \%$, while the MLS-VSX source matches have $\sigma=0.0042 \%$. As the level of agreement is good in both cases, we have confidence in the MLS RRab periods.

\subsection{Comparison with SEKBO RRL Candidates}

The SEKBO survey discovered 2016 candidate RR Lyrae in a survey covering $1675 \mathrm{deg}^{2}$ along the ecliptic (Keller et al. 2008). Like the MLS survey, the SEKBO survey covered a band within $10^{\circ}$ of the ecliptic. However, SEKBO RRL candidate selection was based on the two colors observed simultaneously by the MACHO camera and variability was determined from between 2 and 8 epochs of images. In contrast, the MLS RRab candidates were observed unfiltered and have an average of 130 observations (sampling ranges from 42 to 342 measurements).

We matched the MLS RRab with the SEKBO RRL candidates and found only 103 matches. However, this is not unexpected since the sampling of MLS is highly concentrated within a few degrees of the ecliptic with few observations having been taken near the $\sim 10^{\circ}$ limit. For example, $70 \%$ of the MLS RRab's are within $2^{\circ}$ of the ecliptic compared to $27 \%$ of the SEKBO RRL candidates.

To extend the comparison, we also matched the SEKBO candidates with RRab's from the DR13 sample and the SSS RRab's (G. Torrealba et al. 2013, in preparation). This yielded an additional 538 RRab matches. Because of overlap between the CSS, MLS, and SSS RRab catalogs there were 540 unique RRab stars matching SEKBO RRL candidates. Clearly this number falls far short of the number of SEKBO candidates. 


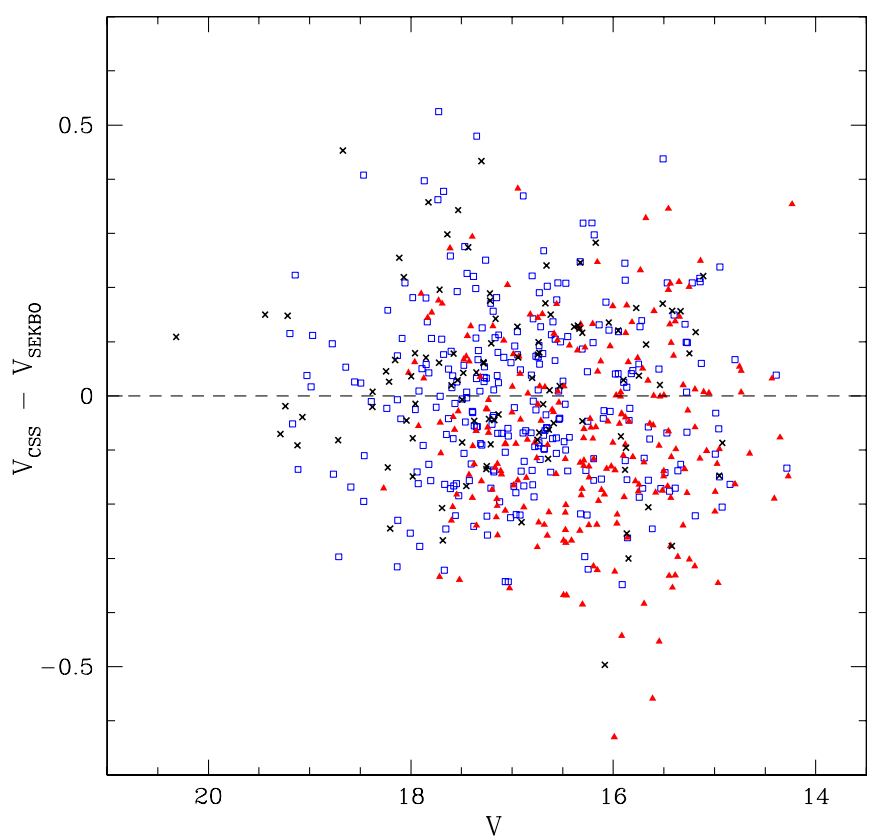

Figure 5. Difference between $V$ magnitudes from CSS and from SEKBO. The black crosses are the MLS matches, the blue boxes are CSS matches, and the red triangles are SSS matches.

(A color version of this figure is available in the online journal.)

We compared average $V$ magnitudes for the Catalina sources with those of the SEKBO sources. In Figure 5, we present the $V$ magnitude differences between the measurements. The average difference between magnitudes is -0.02 mag with $\sigma_{V}=0.16 \mathrm{mag}$, corresponding to a $7.6 \%$ uncertainty in source distances. The internal dispersion for CSS RRab magnitudes from overlapping fields is $\sigma=0.04 \mathrm{mag}$ and thus does not significantly contribute to the observed dispersion.

In order to investigate the SEKBO candidates not found in the Catalina RRab catalogs, we matched the remaining 1481 unmatched SEKBO objects to the SDSS DR8 spectroscopic catalog. We found 40 matches; of these, 35 had measured $\log (\mathrm{g})$ values and 23 had $\log (\mathrm{g})<3.75$ consistent with RRL (DR13). We also matched the SEKBO sources with SDSS DR8 photometry. Of the 276 sources with SDSS photometry, approximately one third had SDSS colors beyond the limits observed for DR13 RRab's. Next, we matched the 1481 SEKBO sources to photometry from MLS, CSS, and SSS surveys. Among the matches, we sub-selected the objects exhibiting significant variability based on the $I_{\mathrm{WS}}>0.6$ and also objects with LS periodic significance $p_{0}<1 \times 10^{-5}$. In total, we find matches for $90 \%$ of the SEKBO objects, including $88 \%$ of the sources that were not selected as RRab's. Combining the known Catalina RRab's and other sources with apparent variability, we find that $74.5 \%$ of the 1833 SEKBO sources we cover exhibit significant variability. In Table 3 , we present the numbers of matches with each of the Catalina surveys, as well as the number of objects selected by our variability and periodicity significance thresholds.

For each of the 1314 SEKBO RRL candidates with Catalina photometry, that were not in our RRab catalogs, we determined an LS period (regardless of their $I_{\text {WS }}$ variability). We inspected both the observed and phase folded light curves of each source and determined a classification based on this photometry. As there are 2759 Catalina light curves matching the 1314 SEKBO candidates, the majority of these sources have multiple light
Table 3

Matches to SEKBO Sources

\begin{tabular}{lcccc}
\hline \hline Survey & RRab & Extra & Variable & Periodic \\
\hline MLS & 103 & 703 & 479 & 192 \\
CSS & 278 & 1046 & 638 & 428 \\
SSS & 237 & 1010 & 460 & 367 \\
Unique & 519 & 1314 & 854 & 668 \\
\hline
\end{tabular}

Notes. Column 1 gives the survey and the combined unique sources from these. Column 2 gives the number of matching RRab catalog sources. Column 3 gives the number of survey sources not within the RRab catalogs. Column 4 gives the number of non-RRab catalog sources exhibiting significant variability $(I>0.6)$. Column 5 gives the number of non-RRab catalog sources exhibiting significant LS periodicity $\left(p 0<1 \times 10^{-5}\right)$.

Table 4

SEKBO RR Lyrae

\begin{tabular}{|c|c|c|c|c|c|c|}
\hline ID & $\begin{array}{l}\text { R.A. } \\
\left({ }^{\circ}\right)\end{array}$ & $\begin{array}{c}\text { Decl. }(\mathrm{J} 2000) \\
\left(^{\circ}\right)\end{array}$ & $\bar{V}$ & $\begin{array}{c}P \\
\text { (days) }\end{array}$ & $\begin{array}{c}A \\
(\mathrm{mag})\end{array}$ & Var Type \\
\hline 128412.1045 & 3.65453 & 3.85789 & 18.30 & & & RRd \\
\hline 103591.297 & 6.04219 & 2.31516 & 17.40 & 0.3044727 & 0.46 & RRc \\
\hline 127806.438 & 6.34742 & -3.25643 & 17.13 & $\ldots$ & $\ldots$ & RRc \\
\hline 116383.660 & 27.46520 & 10.92001 & 17.79 & & $\ldots$ & RRc \\
\hline 117146.1412 & 29.69399 & 12.44214 & 18.60 & 0.3974842 & 0.34 & $\mathrm{RRc}$ \\
\hline 115646.851 & 44.35595 & 15.18618 & 18.35 & & & RRc \\
\hline 115511.269 & 50.62031 & 13.51653 & 16.77 & 0.5326870 & 0.69 & RRab \\
\hline 117289.371 & 64.21176 & 18.87230 & 15.57 & 0.2668644 & 0.42 & $\mathrm{RRc}$ \\
\hline 104856.1587 & 80.69952 & 13.69941 & 17.27 & $\ldots$ & $\ldots$ & $\mathrm{RRc}$ \\
\hline 118386.758 & 110.85990 & 21.62161 & 13.61 & 0.4071660 & 0.15 & RRc? \\
\hline 118692.330 & 120.07333 & 15.15337 & 14.84 & & & RRc? \\
\hline 117700.1008 & 120.99545 & 15.81325 & 15.52 & & & $\mathrm{RRc}$ \\
\hline 117775.379 & 121.92978 & 15.42998 & 15.78 & 0.3299652 & 0.36 & $\mathrm{RRc}$ \\
\hline 118801.2653 & 122.37960 & 15.61271 & 19.15 & 0.4493209 & 1.23 & $\mathrm{RRab}$ \\
\hline 117776.476 & 122.70074 & 16.11215 & 15.36 & & $\ldots$ & RRc \\
\hline
\end{tabular}

Notes. Column 1 gives the SEKBO survey ID. Columns 2 and 3 give the right ascension and declination. Column 4 gives the average magnitude from the AFD fit, if a period is found. Otherwise the weight mean value. Column 5 gives the period. Column 6 gives the amplitude of the AFD fit. Column 7 gives the type of RRL. If a classification is uncertain, a "?" is included.

(This table is available in its entirety in a machine-readable form in the online journal. A portion is shown here for guidance regarding its form and content.)

curves. We discovered 551 additional RRL candidates, 140 eclipsing binary candidates (mainly WUMa), and 140 other types of variable sources (including QSOs, CVs, $\delta$ Scuti variables, and objects where the classification was unclear). This discovery of many non-periodic sources is not unexpected since a number of these are already known, including QSOs and cataclysmic variables. For example, SEKBO 106646.2532 (CSS080623:140454-102702) is a CV that was discovered in outburst by CRTS in 2008 (Kato et al. 2009). Additionally, based on SDSS DR8 spectroscopy, SEKBO-105832.627 is a CV, SEKBO-117146.1412 is a QSO at $z=1.02$, and SEKBO-096514.1360 is an eclipsing WD-MD binary system (Rebassa-Mansergas et al. 2010).

In order to obtain the best periods for the clear RRL, and to find RRL that may have been missed during our inspection, we ran every light curve through the AFD software (G. Torrealba et al. 2013, in preparation). For 307 of the 551 RRL candidates we found good Fourier fits and periods consistent with RRab's and RRc's. In Table 4, we present all the new SEKBO RRL found using Catalina data (including RRL candidates where the 
period is uncertain). This table contains $263 \mathrm{RRab}, 282 \mathrm{RRc}$, and $11 \mathrm{RRd}$ candidates.

Combining the new RRab's with the 540 from Catalina catalogs we find that $\sim 36 \%$ were missing from our catalogs in good agreement with the simulations presented in DR13. The overall sample suggests that $24 \%$ of the RRL are RRc stars whereas Keller et al. (2008) estimated that $\sim 10 \%$ of the SEKBO candidates would be RRc's. However, we note that Pietrukowicz et al. (2012) found 30\% RRc among their $\sim 15,000$ bulge RRL. Nevertheless, it is possible that some fraction of the RRc candidates we identify may be W UMa variables (due to the similarity of their light curves). The uncertain separation of RRc's and W UMa types is the main reason why RRc's are usually excluded as distance indicators.

Our results suggest that $60 \%$ of the SEKBO RRL candidates are likely RRL based on the 1833 objects covered by Catalina data. This is $2.2 \sigma$ smaller than the $24 \pm 7 \%$ non-RRL contamination estimated by Prior et al. (2009b). However, we note that the Prior et al. (2009b) sample was based on an average of just nine photometric measurements for $106(\sim 5 \%)$ of the SEKBO stars. Prior et al. (2009a) followed 21 additional SEKBO RRLs overlapping the Sgr tidal stream. More recently, Akhter et al. (2012) followed 137 SEKBO candidates and found 57 to be RRLs, although they found a high fraction with colors matching the colors of RRL candidates from SDSS data.

From Catalina photometry we have an average of 121 observations per light curve (250 per object) covering $90 \%$ of the SEKBO sources. Our analysis suggests that $\sim 25 \%$ of the SEKBO sources are either not variable or have significantly less variability than expected for RRL. Nevertheless, with $>1000$ likely RRL from the SEKBO survey, the results shows that large numbers of RRL can be found using very small numbers of observations and color selection.

\section{OUTER HALO RR LYRAE}

The outer halo RRL discovered in this analysis overlap with the tidal stream first identified by Newberg et al. (2003) using photometrically selected BHB candidates. As noted above, additional BHB candidates have since been found in SDSS DR5 (Newberg et al. 2007) and SDSS DR7 data (Ruhland et al. 2011). Furthermore, Ivezic et al. (2004) discovered many RRL candidates overlapping the Sgr stream plane. The most distant sources coincide with the Newberg et al. (2003) BHB candidates. However, the Ivezic et al. (2004) RRL sample was based on a photometric selection combined with limited variability information. Of the 1269 Ivezic et al. (2004) RR Lyrae candidates selected in the SDSS stripe-82 region, only $483(38 \%)$ were eventually confirmed by Sesar et al. (2010). It seems likely that a similar fraction of the sources found in this marginally significant $(2 \sigma-3 \sigma)$ detection by Ivezic et al. (2004) were indeed RRL.

Recently, additional evidence for a distant group of RRL stars was found by Sesar et al. (2012). These authors discovered eight $\mathrm{RRab}$ between $124^{\circ}<\alpha<133^{\circ}$ and $18^{\circ}<\delta<24^{\circ}$ at heliocentric distances $77<d_{H}<96 \mathrm{kpc}$. This region lies on the edge of the fields where BHB candidates were previously discovered by Newberg et al. (2003). Sesar et al. (2012) selected potential RRab candidates based on variable Palomar Transient Factory (PTF) sources with RRL colors based on SDSS DR8 photometry. Using simulations Sesar et al. (2012) estimated that in their worst-case scenario their search recovered $95 \%$ of their simulated sources with at most one RRab star being missed. Six of the Sesar et al. (2012) RRab's were independently discovered

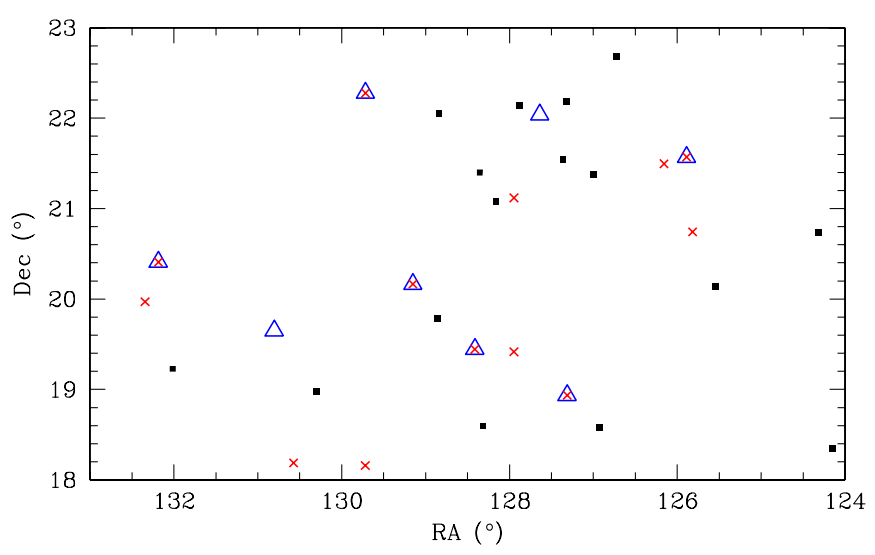

Figure 6. Distribution of RRab within the PTF Praesepe region. The triangles show the locations of Sesar et al. (2012) RRab's, while the crosses mark RRab's found by MLS with $76<d_{H}<97 \mathrm{kpc}$. The dots mark the locations of RRab's with $d_{H}<76 \mathrm{kpc}$.

(A color version of this figure is available in the online journal.)

in our searches, while the remaining two were missed due to poor sampling. For all six known RRab, we find periods matching those given by Sesar et al. (2012) to better than $0.04 \%$.

In addition to the six PTF RRab, we have discovered seven $\mathrm{RRab}$ within the region and distance range noted by Sesar et al. (2012). We find 16 more RRab's in this region at heliocentric distances $d_{H}<70 \mathrm{kpc}$. As we missed two of the PTF RRab's it is possible that there are even more RRab's within this region. In Figure 6, we present the locations of the RRab's found within the PTF Praesepe fields. Among the set of eight RRab's, Sesar et al. (2012) found tentative evidence for two separate groups of RRL based on radial velocities. One group of four RRab's was noted as having radial velocities in the Galactic standard of rest of $\langle\mathrm{Vgsr}\rangle=16 \pm 7 \mathrm{~km} \mathrm{~s}^{-1}$, while the other had $\langle\mathrm{Vgsr}\rangle=78 \pm 6 \mathrm{~km} \mathrm{~s}^{-1}$. Although Sesar et al. (2012) note that there is a $37 \%$ chance that these stars are drawn from the same Gaussian velocity distribution based on the Shapiro \& Wilk (1965) SW statistic, they assume that their eight sources are representative of the overall velocity distribution and via numerical simulations find a $<0.6 \%$ chance that the sources would exhibit the observed velocities. These authors also discovered that the metallicities of the two groups were consistent to within uncertainties.

In total, we identify 103 RRab's at heliocentric distances beyond $70 \mathrm{kpc}$. Four of these RRab's appear near $\alpha=355^{\circ}$, $d_{H}=85 \mathrm{kpc}$. These stars are associated with the Pisces stream (Sesar et al. 2007; Watkins et al. 2009). However, we see no evidence for variations in distance in agreement with the results of Kollmeier et al. (2009). This supports evidence that this system is not part of a tidal stream itself. Of the remaining stars, 82 RRab's lie within the range $100^{\circ}<\alpha<150^{\circ}$ and $14^{\circ}<\delta<30^{\circ}$. These objects exhibit a range of distances and thus comprise significant evidence for a stream of stars, suggesting association with a tidally disrupted galaxy.

To better understand the nature of the relation between the distant RRab's and the Sgr stream we selected the RRab sources within $11^{\circ}$ of the Sgr plane (as defined by Majewski et al. 2003). Based on Koposov et al. (2012) and DR13, most Sgr stream stars, including RRab's, lie within these limits. We combine RRab's from DR13 along with new CSS RRab's and MLS RRab's in the range $145^{\circ}<\alpha<210^{\circ}$ within the Sgr stream region. We transform the RRab distances to Galactocentric values and plot the sources in Figure 7. In this plot the distant stream of RRab's 


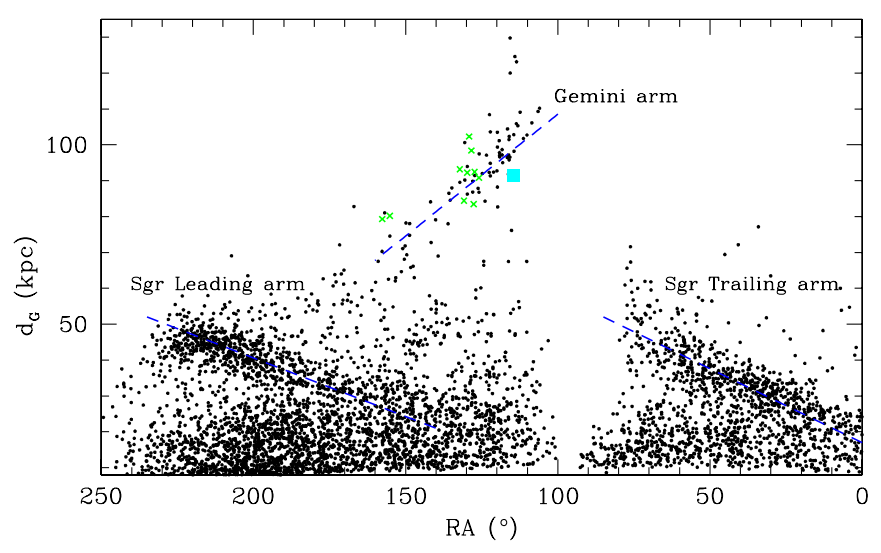

Figure 7. Galactocentric distance distribution for stars within $11^{\circ}$ of the Sagittarius plane. Large dots show the MLS and CSS RRab data. The crosses show PTF RRab's from Sesar et al. (2012). The dashed line presents a simple linear fit to the RRab with $d_{G}>70 \mathrm{kpc}$. The large cyan square presents the location of globular cluster NGC 2419.

(A color version of this figure is available in the online journal.)

becomes clearer, as do the leading and trailing Sgr streams that are observed between $150^{\circ}<\alpha<250^{\circ}$ and $10^{\circ}<\alpha<70^{\circ}$, respectively.

Among the set of distant MLS RRab's, the four most distant objects are found within the $\sim 4 \mathrm{deg}^{2}$ region $113.5<\alpha<$ $115.7,22.8<\delta<24.8$. These RRab's have distances $119.9<d_{G}<129.7 \mathrm{kpc}$, their average magnitudes varying by $\sim 0.2 \mathrm{mag}$, and are consistent with no difference at their observed brightness. This concentrated group is significantly fainter than any of the other MLS RRab's, suggesting a possible association between them that varies from that of the other sources. However, measurements of radial velocities and metallicities are required before we can rule out association with the other RRab's.

After excluding the group of four very distant RRab's, and four outlier RRab's near $d_{G} \sim 70 \mathrm{kpc}$ with $\alpha \sim 115^{\circ}$ we determine the slope of the outer stream by selecting the RRab's with $60<d_{G}<115 \mathrm{kpc}$, in the range $100^{\circ}<\alpha<160^{\circ}$. A simple linear fit gives $d_{G}=177( \pm 5)-0.68( \pm 0.04) \times \alpha$. The slope of this line suggests that the distant stream does not meet with the so-called trailing Sgr stream lying in the region $0^{\circ}<\alpha<80^{\circ}$.

In order to better visualize the direction and slope of the streams, in Figure 8 we present a polar plot of the same RRab sources in the Sgr plane coordinate system. In contrast to Figure 7, here we plot average static star magnitudes for the RRab's fainter than $V=16.5\left(d_{H}>15 \mathrm{kpc}\right)$. Clearly a more accurate description of the path of this outer stream requires more RRab's in the region $\Lambda>240^{\circ}$, which is not covered by the MLS photometry. As noted earlier, many new CSS RRab's were found in this region. However, as shown in Figure 2 and DR13, RRab's fainter than $V=19.5\left(d_{G}>60 \mathrm{kpc}\right)$ are at the limit of CSS data. Discoveries in CSS data covering this region will be likely biased to the brighter, nearer sources.

To further explore the relationship to the Sgr stream, we plot the RRab's in the Sgr $X-Y$ plane system of Majewski et al. (2003) in Figure 9. Here we mark the proposed path of the Gemini stream. We also plot the Law \& Majewski (2010) $N$-body model of the $\mathrm{Sgr}$ stream system. The model is a reasonable match to the structure of the inner RRab data. The agreement is increased if the source distances are reduced by $\sim 11 \%$. However, as found by Ruhland et al. (2011) the Gemini

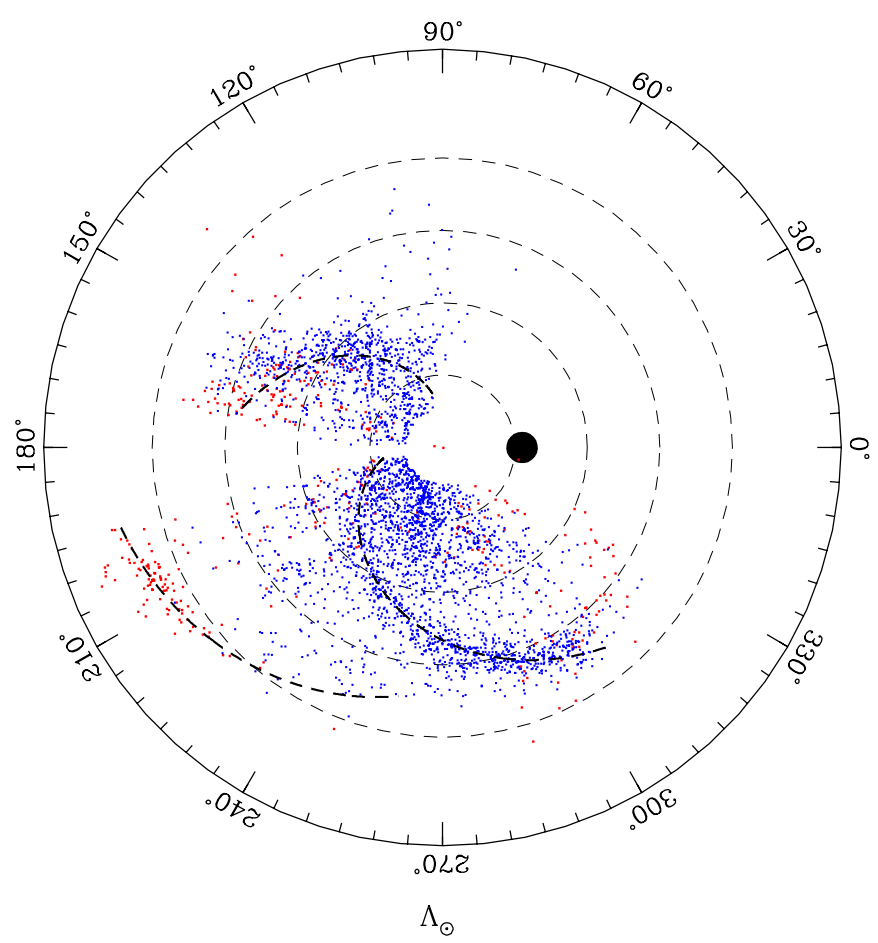

Figure 8. Polar plot of the magnitude distribution of MLS (red points) and CSS (blue points) RRab stars in the Majewski et al. (2003) Sgr coordinate system. The dashed circles present $V$ magnitudes of 17, 18, 19, and 20. The three bold arcs show the approximate average magnitudes of the three Sgr streams. The large dot shows the location of the Sgr dwarf galaxy.

(A color version of this figure is available in the online journal.)

stream is not explained by this model or other models of the Sgr stream system. Nevertheless, it appears that if the path of Sgr leading debris was extended, it might better match the location of the sources. Indeed, Law \& Majewski (2010) found that if Sgr had been orbiting 2 Gyr longer, a much longer leading arm would be present. Nevertheless, their simulations did not produce sources corresponding to the distances and locations of the BHB candidates and RRL. It is apparent that the Sgr trailing arm model is a very poor match to the Gemini stream, both in extent and location.

\subsection{Feature Significance}

To determine the significance of the Gemini stream it is necessary to compare the source density with that expected from halo models. We adopt the halo density model from Sesar et al. (2010) for our comparison. The local density of RRL is determined using the $N$ th nearest neighbor method. We find the eighth nearest star to the point where we want to calculate the density and calculate the area that bounds these stars. We use eight stars motivated by Ivezic et al. (2005) who found that this number gives the best results for the underlying density on their improved Bayesian method. We also saw that this number was a good combination of precision and computational efficiency.

We compute the observed number density within our grid $(r)$ and density from the Sesar et al. (2010) halo model $\left(r_{m}\right)$. To visualize the overdensities on the Sgr plane, we produce a $100 \times 100$ grid on the Majewski et al. (2003) Sgr coordinates at $Z=0$ (for the $X-Y$ plot) and $B=0$ (for the $\Lambda-D_{\text {Sgr }}$ plot). In Figure 10 we plot the resulting density ratios in the $\Lambda-D_{\mathrm{Sgr}}$ system and in Figure 11 we plot the densities in the $X-Y$ plane of the Sgr system. The densities here can be readily contrasted sources in Figure 9. 

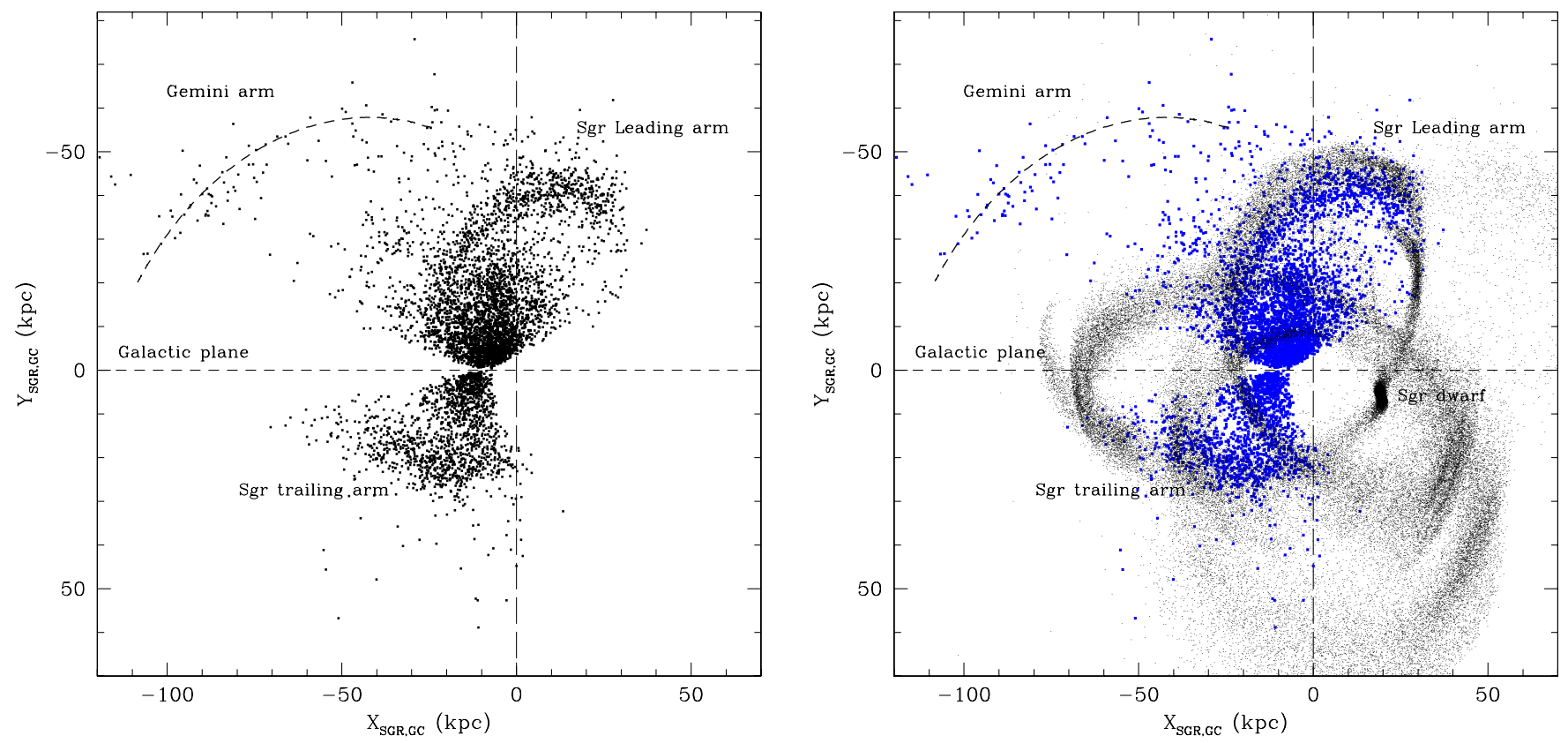

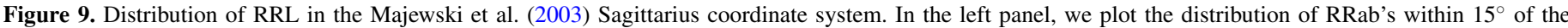
Sagittarius plane. In the right panel, we plot the RRab's along with the Law \& Majewski (2010) Sagittarius streams $N$-body model.

(A color version of this figure is available in the online journal.)

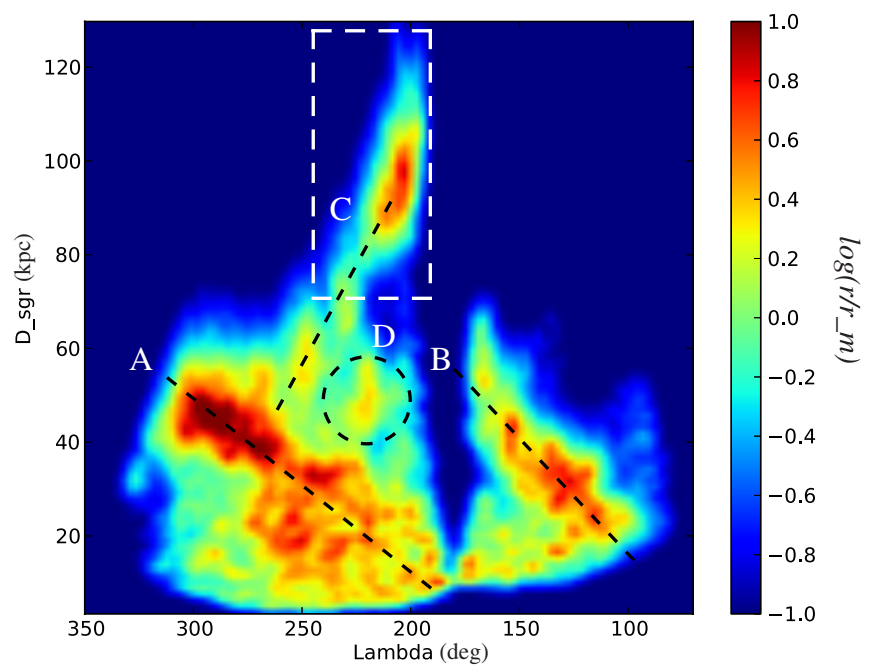

Figure 10. Halo subtracted density distribution of RRab stars in Majewski et al. (2003) Sagittarius polar coordinate system. (A) The location of RRab's in the Sgr leading arm. (B) The RRab's in the Sgr trailing arm. (C) The proposed location of the Gemini stream. (D) The location of another possible overdensity of RRab's. The region selected for significance testing is outlined by the longdashed line.

(A color version of this figure is available in the online journal.)

The main density features are the leading and trailing Sgr arms (marked by A and B, respectively). The Gemini stream (marked as $\mathrm{C}$ ), largely has a higher density ratio than the trailing stream system (marked by B). Another possible feature is seen near $\Lambda=220, D_{\mathrm{Sgr}}=50 \mathrm{kpc}($ marked $D)$. This has much lower significance than the other features, yet is located where models predict the Sgr trailing arm should cross the Galactic plane and meet with the trailing arm $B$. Since the Gemini feature, like much of the Sgr stream, is offset from the Sgr plane, we have collapsed the source $\mathrm{Z}$ positions to visualize the stream.

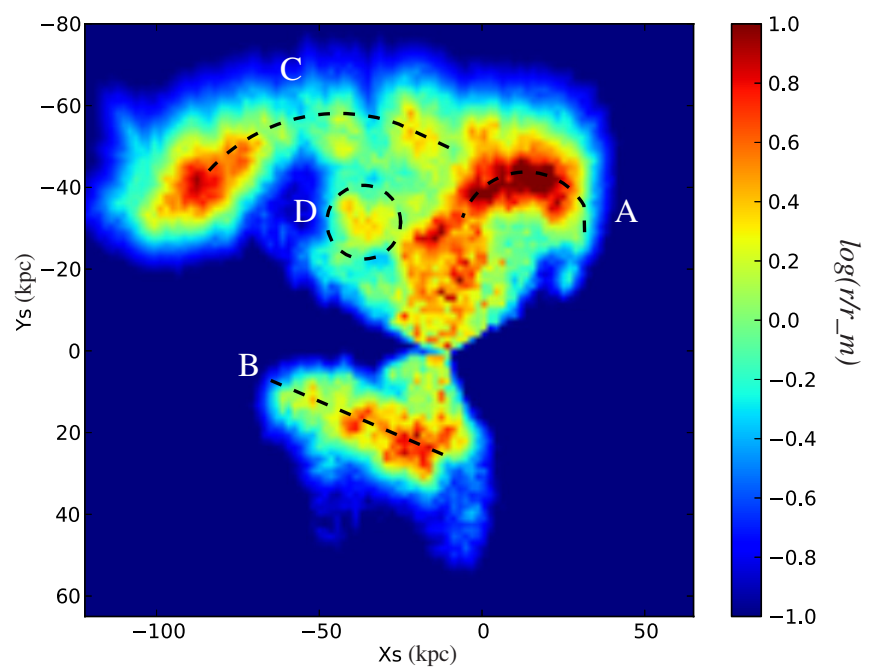

Figure 11. Halo subtracted distribution of RRab stars projected to the $X-Y$ plane Majewski et al. (2003) Sagittarius coordinate system. (A) The location of RRab's in the Sgr leading arm. (B) The RRab's in the Sgr trailing arm. (C) The proposed location of the Gemini stream. (D) The location of another possible overdensity of RRab's.

(A color version of this figure is available in the online journal.)

Unlike the Gemini stream, the leading and trailing Sgr arms A and B are already well known (Law \& Majewski 2010, and references therein). To quantify the significance of the Gemini stream, we estimated the number of stars expected by the model at the position of the stream. For simplicity, we selected the volume that bounds the stream in the Sgr coordinate system; that is, $190<\Lambda<240,1<\mathrm{B}<17$, and $70<R_{\text {Sgr }}<130$. Based on the Sesar et al. (2010) model density, within this volume we would expect to find 373 RRL assuming $100 \%$ detection efficiency. However, only 106 RRL were found. Assuming pure Poisson uncertainties, this suggests the Gemini area has $\sim 14 \sigma$ underdensity. As our detection efficiency is close to $70 \%-80 \%$ 

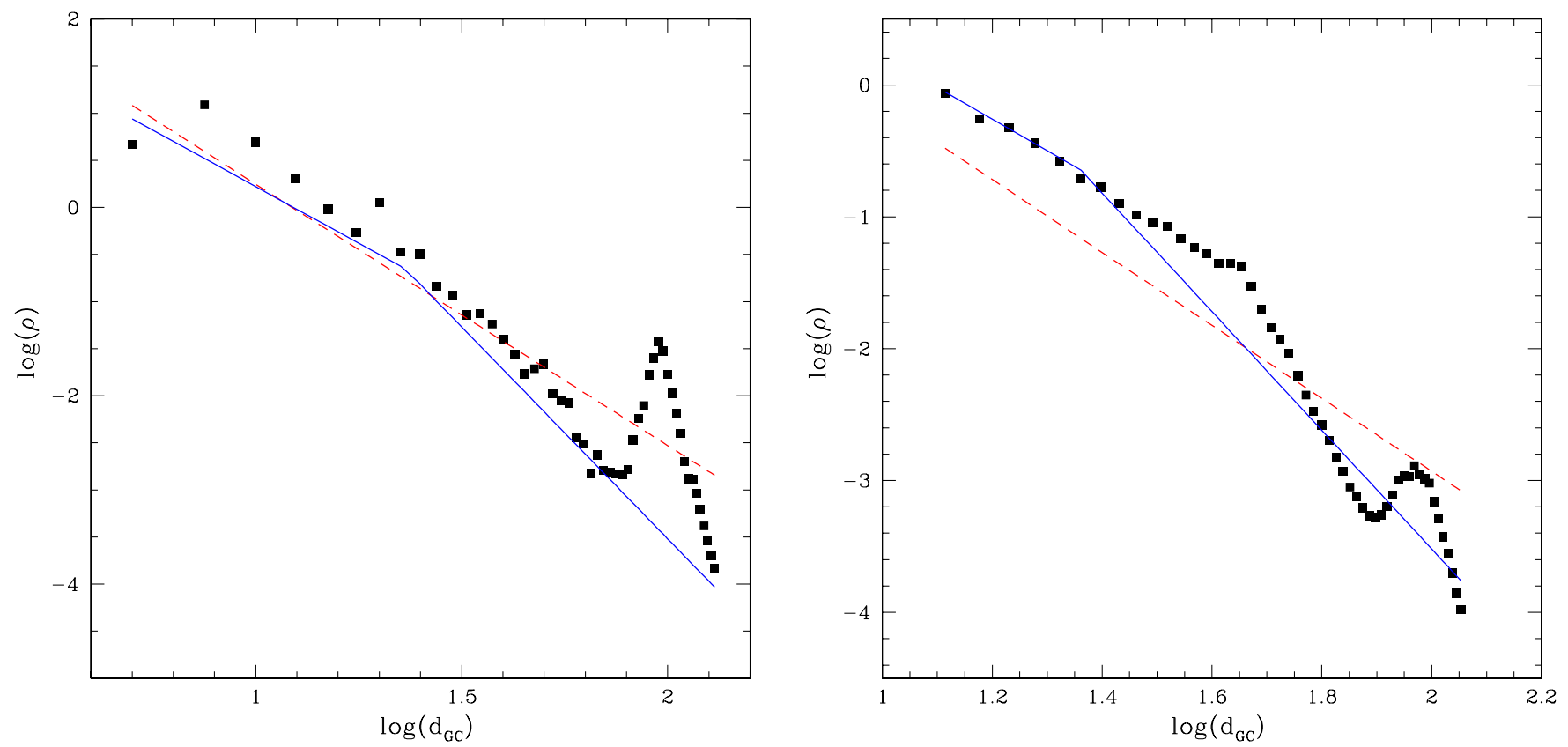

Figure 12. Halo density of RRL. Here we plot the observed density of RRL compared with the Sesar et al. (2010) halo model (dashed line) and the adjusted Watkins et al. (2009) model (solid line). In the left panel, we plot the values for the Sgr along the line $\Lambda=210^{\circ}$. In the right panel, we plot the values averaged over Sgr stream region.

(A color version of this figure is available in the online journal.)

based on DR13 this short fall cannot be explained purely by missing RRL. The detection of no distant RRL across most of the MLS survey area strongly suggests that the halo is much less dense than expected by the model. This result is in agreement with the results of Watkins et al. (2009) and Sesar et al. (2010) based on halo RRL discovered in SDSS stripe-82. This confirms that the halo density declines more rapidly than suggested by the Juric et al. (2008) halo model.

As a second model comparison, we compare the RRL densities with the Watkins et al. (2009) halo model. Their results suggest a break in density occurs $d_{G C}=23 \mathrm{kpc}$. Matching the model to the data we find that the Watkins et al. (2009) model requires normalization by a factor of 11 to match our data. This is in good agreement with the factor of 10 found by Sesar et al. (2010). In Figure 12, we plot the observed radial density compared with the Sesar et al. (2010) model and the normalized Watkins et al. (2009) model. The observations are found to be in good agreement with the Watkins et al. (2009) model, although the break in density appears to occur nearer to $50 \mathrm{kpc}$ when averaged over a large area. However, this is because the densities are enhanced by RRL in the Sgr leading and trailing arms. Based on the Watkins et al. (2009) model we expect to find $50 \mathrm{RRL}$ in the region selected above. The number of Gemini stream RRL we find is thus $7.9 \sigma$ larger than expected from this model.

\subsection{RR Lyrae Populations}

To investigate differences between the Gemini RRab sources and the overall population, one can infer the Oosterhoff type based on the RRab period-amplitude relationship (Smith et al. 2011 and references therein). However, since average colors were used to transform the MLS light curves, we need to account for the effect of color variation of the RRab light curves. In DR13 we found that RRab amplitudes were systematically reduced by 0.15 due to pulsational color variations within the broad bandpass of CSS images compared to $V$-band. We also found that the CSS Oosterhoff type-I (OoI) RRab's exhibit a well-defined amplitude limit that is $0.1 \mathrm{mag}$ higher than the Zorotovic et al. (2010) period-amplitude relationship.

To separate OoI RRab candidates from Oosterhoff type-II's (OoII's), we correct the MLS amplitudes to values by adding 0.15 mag. Next we define a set of RRab's that are between 0.1 and $0.25 \mathrm{mag}$ above the average OoI period-amplitude relationship. These objects are a mixture of Oosterhoff types (although some may be Oosterhoff intermediate sources) that we remove from consideration. We then investigated the variation in MLS RRab's Oosterhoff types with distance. In Figure 13, we plot the period-amplitude distribution of the MLS RRab's.

By dividing these sources into a nearer sample consisting of RRab with $70<d_{G}<95 \mathrm{kpc}$ and the more distant RRab $d_{G}>95 \mathrm{kpc}$ we see a division in types. In Figure 13, we also plot the period-amplitude distribution for the Gemini RRab's. We also overplot the Zorotovic et al. (2010) OoI line and an OoII line that is offset by +0.07 in $\log (P)$ to match the CSS observations. The nearer RRab's exhibit a mixture of Oosterhoff types when compared to the Zorotovic et al. (2010) period-amplitude relationships. This is significant evidence that the RRab's in the range $70<d_{G}<95 \mathrm{kpc}$ do not come from a single population. In contrast, the distant MLS RRab's all lie near the OoI line, suggesting that they come from a single population that is more metal-rich.

In Figure 14, we show the locations of Gemini stream RRab's after removing the MLS RRab's with ambiguous Oosterhoff type. The CSS RRab's with $70<d_{G}<95 \mathrm{kpc}$ have also been included, but are not separated by Oosterhoff type since their amplitudes and periods are less certain than the MLS RRab's. This figure shows that the Gemini RRab's are spread across the width of the Sgr stream system. It also shows that the OoII RRab's selected among the nearer RRL set $\left(70<d_{G}<95 \mathrm{kpc}\right)$ are distributed across the region. The nearer OoI group ends around $\alpha \sim 125^{\circ}$ where the stream divides between the nearer 

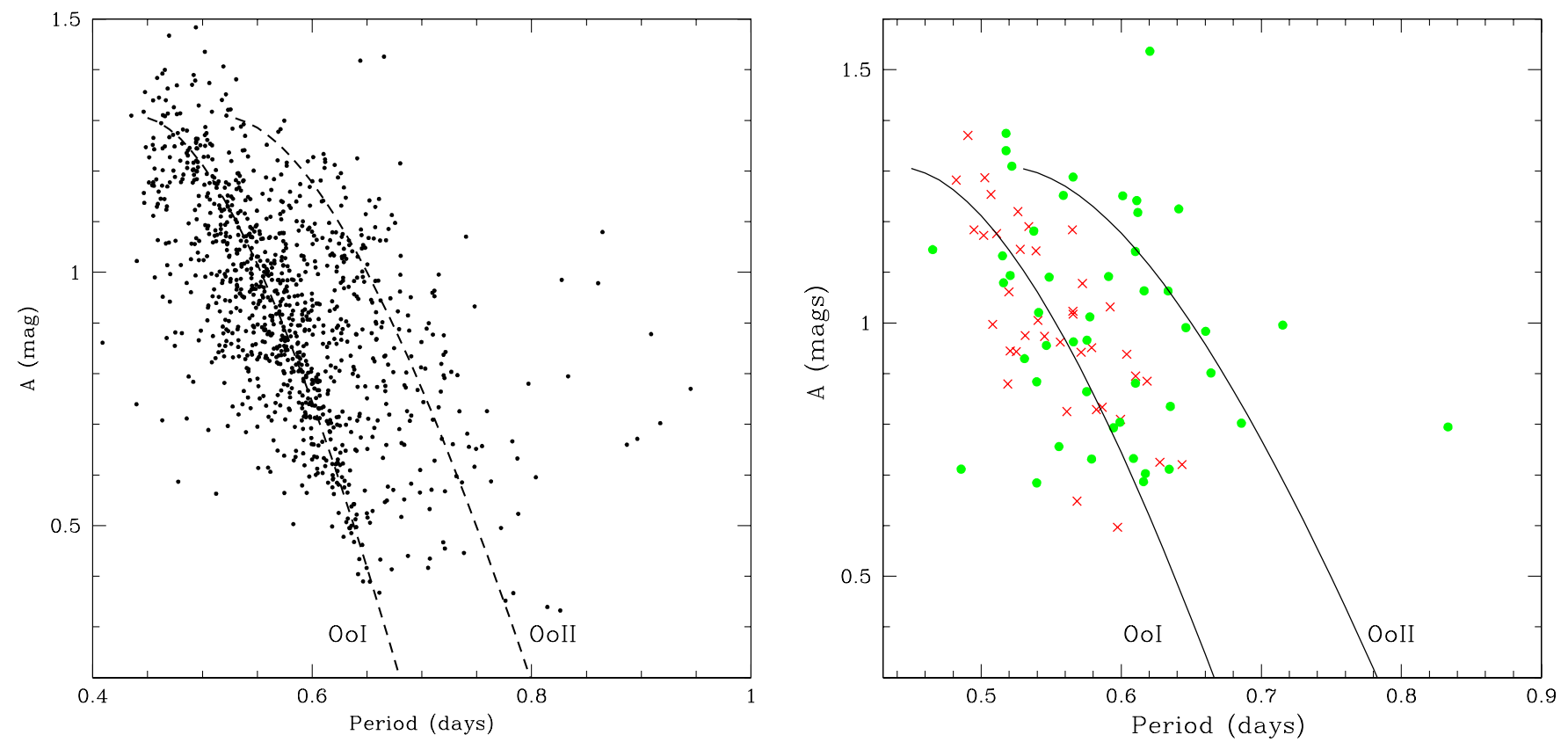

Figure 13. Period-amplitude distribution of RRab stars. In the left plot we present the period and amplitudes for all the MLS RRab's. In the right plot, the MLS RRab's with $70<d_{G}<95 \mathrm{kpc}$ are plotted with green dots, and those with $d_{G}>95 \mathrm{kpc}$ are red crosses. The solid lines mark the Zorotovic et al. (2010) period-amplitude relationships for OoI and OoII and the dashed lines outline a region where the RRab Oosterhoff types are mixture of OoI and OoII. shown. In both plots the RRab $V$-band amplitudes plotted have been increased by 0.15 mag to account for color variations in the RRab's between maximum and minimum light.

(A color version of this figure is available in the online journal.)

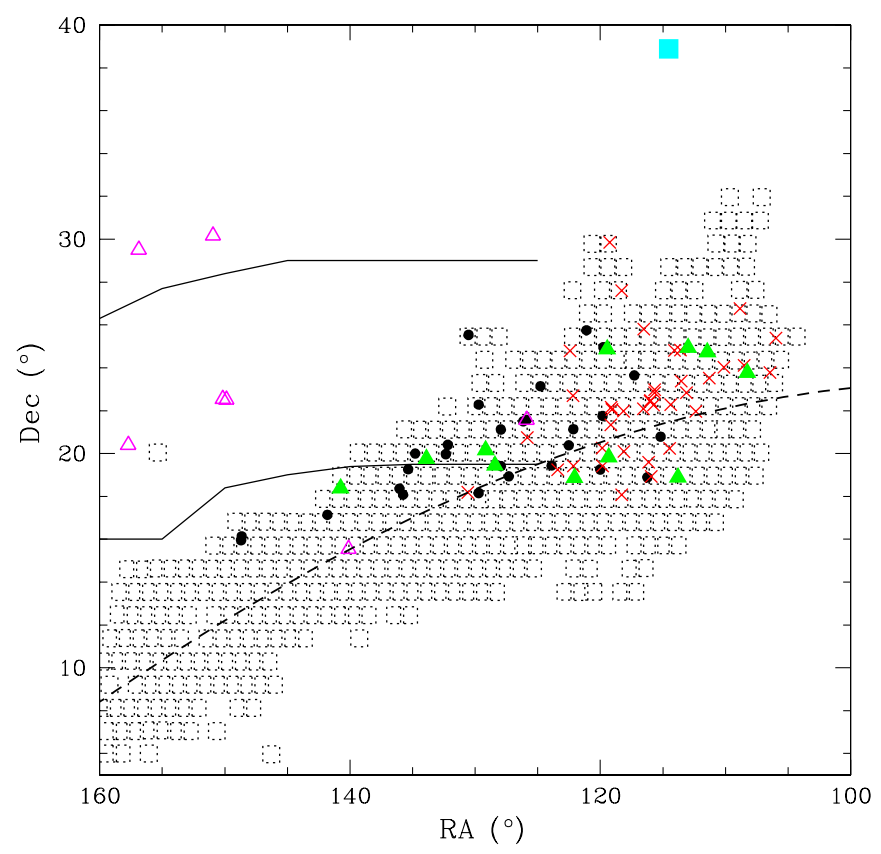

Figure 14. Locations of distant halo RRab's. Here we present the locations of RRab's relative to the MLS survey fields marked by the boxes. The RRab with $70<d_{G}<95 \mathrm{kpc}$ and periods and amplitudes most consistent with OosterhoffI (i.e., Sagittarius dSph-like) sources are marked with large dots. The MLS RRab in this distance range that are most consistent with an Oosterhoff-II type (i.e., NGC 2419-like) classification are given by filled green triangles. The CSS RRab's with $70<d_{G}<95 \mathrm{kpc}$, but uncertain Oosterhoff types are marked with open magenta triangles. The MLS RRab's with $d_{G}>85 \mathrm{kpc}$ are given by red crosses. Here the short-dashed line shows the location of the ecliptic plane. The large cyan square shows the location of NGC 2419. The solid lines shows the rough locations of two Sgr streams given by Belokurov et al. (2006).

(A color version of this figure is available in the online journal.) and farther groups. Analysis of this figure suggests that there may be two or more overlapping populations: one that follows a steep distant gradient and is predominantly OoI, and another that has a shallower gradient and is an OoII population. With respect to this figure, we once again note that the MLS RRL are naturally concentrated toward the ecliptic because of sampling, so it is not possible to make inferences about changes in density across the Sgr stream.

\subsection{Comparison with SDSS Data}

While the SDSS photometry has little of the repeated photometry required to unambiguously identify RRab's, it is deeper than both CSS and MLS data (reaching HB stars to $g \sim 22$ ). Additionally, SDSS data do cover most of the Gemini stream observed in the MLS data as well as the main region where MLS data do not overlap the Sgr stream. Therefore, it is possible to use these data to bridge the gap in between the depth of CSS data and coverage of MLS photometry.

Unlike previous authors who searched for BHB stars covering the Sgr tidal streams, here we sought to select both BHB and potential RR Lyrae stars. Based on the colors of the distant MLS RRab's and prior BHB work, we investigated the observed colors of RRL in SDSS photometry and selected SDSS DR8 stars within $0.95<(u-g)_{0}<1.5,-0.2<(r-i)_{0}<0.2$, $-0.35<(g-r)_{0}<0.22$, SDSS object type $=6$ (star), and $17<g_{0}<22$. Based on DR13 we know that the bulk of RRab's are located near $(g-r)_{0}=0.25,(r-i)_{0}=0.1$ and $(i-z)_{0}=$ 0.05 . However, RRab's with these colors are outnumbered by MSTO stars by a factor of $>100$ (Koposov et al. 2012). Using maps of SDSS source density, we sub-selected stars in the range $2.7 \times(r-i)_{0}+0.25>(g-i)_{0}>2.7 \times(r-i)_{0}-0.1$ for $(g-i)_{0}<0.05$. This selection retains most of the BHB candidates of Ruhland et al. (2011) as well as $22 \%$ of the distant MLS RRab sample and many BS stars. 


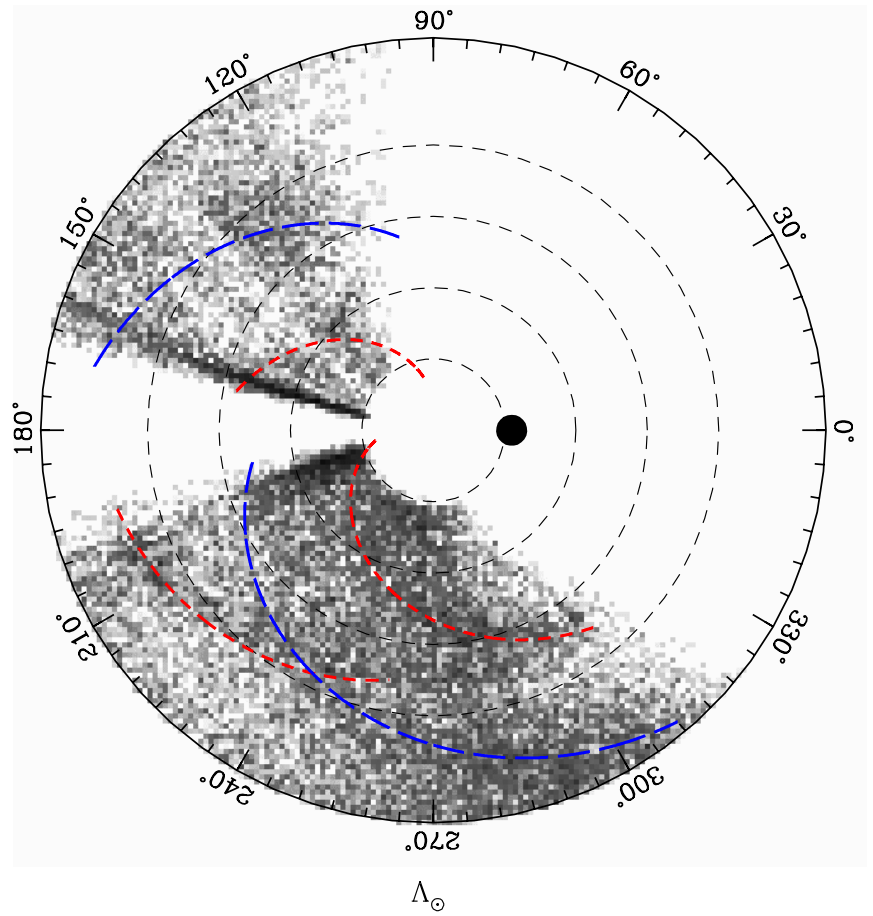

Figure 15. Spatial point-density distribution of SDSS HB candidates near the plane of the Sgr streams $\left(-11^{\circ}<B<11^{\circ}\right)$ with magnitudes $17<V<21.5$. The locations of RRab streams from Figure 8 are presented with short-dashed lines while the expected locations of BS streams which mirror the HB streams are shown with long-dashed lines.

(A color version of this figure is available in the online journal.)

This color selection reduces the initial number of SDSS point sources in our selection from 1.65 million to 81,552 . By selecting stars in the Sgr stream region, with $-11^{\circ}<\Lambda<11^{\circ}$, the number reduces to $23,507 \mathrm{HB}$ candidates. To match the MLS photometry we transform the SDSS photometry to $V$ magnitudes using Ivezic et al. (2007). As with Newberg et al. (2003, 2007) and Ruhland et al. (2011) we found the spatial distribution of SDSS HB candidates suffers from a significant crowding and background that is best viewed in source density. In Figure 15, we provide the HB candidates in the form of the Hess (pointdensity) diagram. This figure shows evidence that the density of HB candidates closely follows the fit to the distant Gemini stream of RRab's, in agreement with the distribution of BHB candidates from Ruhland et al. (2011) and others. Here the scaling has been set to match that in Figure 8 where the main streams are visible. In addition to the RRL, the paths expected for BS stars that pass the color cuts and form a shadow that is $\sim 2$ mag fainter than the HB stars is also shown.

To obtain another view of MLS RRL in the Gemini stream in relation to the SDSS HB candidates, we divided the SDSS sources into three groups. These were bright sources, with $17<V<18.5$, intermediate-brightness sources, with $18.5<$ $V<19.8$, and faint sources, with $19.8<V<20.7$. In Figure 16, we present the locations of these sources. The bright sources were selected to show the nearby HB stars as well as the BS stars in the Monoceros stream (near the Galactic anticenter at $\alpha=110^{\circ}-120^{\circ}$ limit of the SDSS coverage). The intermediate brightness sources were selected to be indicative of the HB stars in the leading arm of the Sgr stream. The faintest sources were selected to include the $\mathrm{HB}$ candidates in the Gemini stream. However, this also includes BS stars that mirror the distribution of HB stars along the Sgr leading arm.

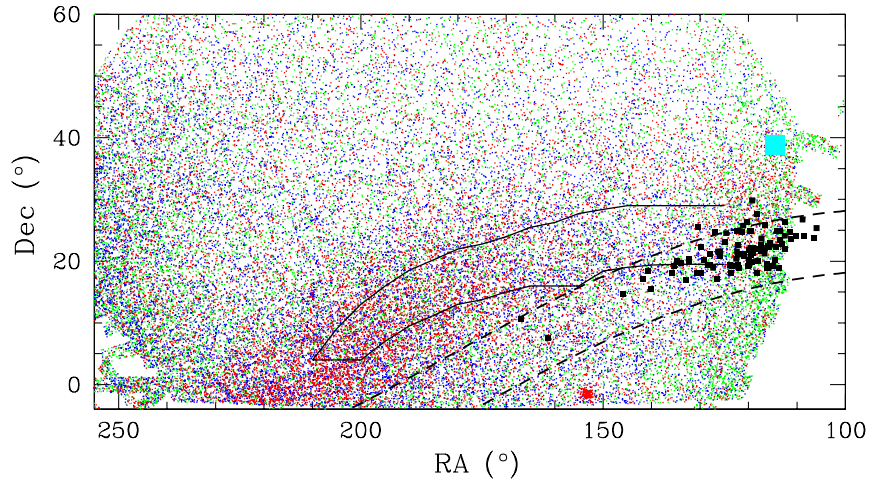

Figure 16. Distant MLS RRL and SDSS HB candidates overlapping the Sgr tidal streams. The green points show SDSS stars with $17<V<18.5$, the blue points those with $18.5<V<19.8$, and the red points those with $19.8<V<20.7$. The black squares show the locations of RRL with $d_{G}>80 \mathrm{kpc}$, and the large cyan box shows the location of NGC 2419. The dashed lines connect points with $b=-10^{\circ}$ and $b=10^{\circ}$. These are the approximate limits of the MLS survey. The solid lines show the locations of two Sgr streams given by Belokurov et al. (2006).

(A color version of this figure is available in the online journal.)

The figure clearly shows the overdensity associated with the Sgr streams system. The separation into two streams is not clear here since there are far fewer BHB stars than the MSTO stars used by Belokurov et al. (2006).

\subsection{SDSS Spectra of RRL Sources}

We matched the entire MLS RRL catalog with the SDSS DR8 spectroscopic catalog and found 89 matches. A much larger sample of RRL with spectra is given in DR13. However, here our main purpose was analysis of the distant halo RRL. We found that 16 of these matches are sources beyond $70 \mathrm{kpc}$. As noted earlier, many of the faint RRL candidates were found based on their SDSS spectra. The 16 RRL with SDSS spectra include 12 RRab's and four RRc's.

To separate the radial velocities from the velocities due to pulsation, we use the SDSS observation times and the Fourier fits to derive the phase at which the RRL spectrum was observed. As noted in DR13, for SDSS spectra, radial velocities are determined by averaging both Balmer and metallic lines (mainly Ca lines). We follow DR13 by applying Sesar (2012) velocity corrections for pulsation based corrections derived from both hydrogen and metallic lines. The average velocity correction for the $12 \mathrm{RRab}$ is $13 \mathrm{~km} \mathrm{~s}^{-1}$, in good agreement with the uncertainty derived from 905 RRab spectra in DR13 $\left(\sigma=14.3 \mathrm{~km} \mathrm{~s}^{-1}\right)$. We adopt this level of uncertainty for all the SDSS RRab spectra.

For the four RRc stars that pulsate in the first overtone mode the velocity of the pulsation is much smaller than for the RRab's. Based on the RRc's observed by Liu \& Janes (1989) and Jones et al. (1988), the amplitude is expected to be approximately $20 \mathrm{~km} \mathrm{~s}^{-1}$. To account for this factor, for these RRL we increase the observed SDSS radial velocity uncertainties by an additional $10 \mathrm{~km} \mathrm{~s}^{-1}$. Following Law \& Majewski (2010) we transform the radial velocities to the Galactic standard of rest assuming a solar peculiar motion of $(\mathrm{U}, \mathrm{V}, \mathrm{W})=(9,12+220,7) \mathrm{km} \mathrm{s}^{-1}$ in the Galactic Cartesian coordinate system.

In Figure 17, we plot the radial velocities for the outer halo MLS RRab's with spectra, the CSS RRab's with $d_{G}>40 \mathrm{kpc}$, and the 10 PTF RRab's found by Sesar et al. (2012). We also plot the velocities predicted by Law \& Majewski (2010) N-body simulations. The figure shows that two of the RRab's observed 


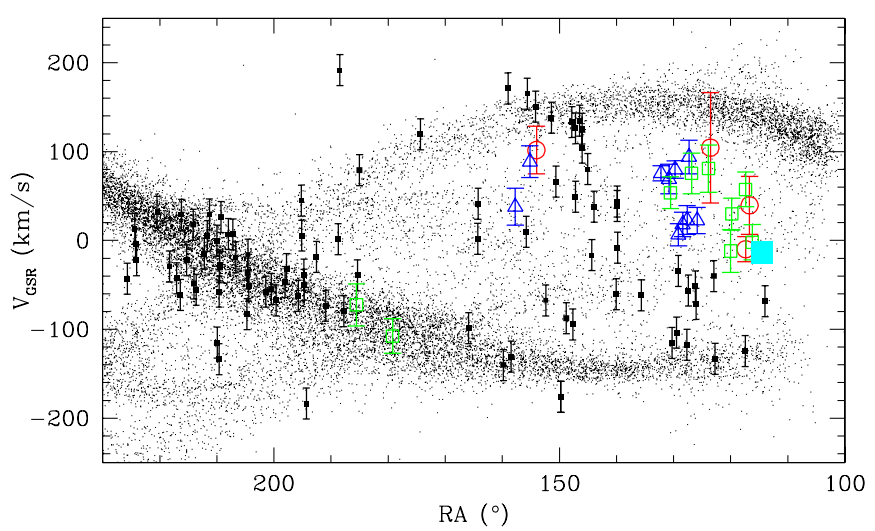

Figure 17. Radial velocities for outer halo RR Lyrae. The blue triangles are values for Sesar et al. (2012) "Cancer Group A" and "Cancer Group B" RR Lyrae. Green squares and red circles are MLS RRab and RRc measurements, respectively. The dots present points from the Law \& Majewski (2010) Sgr $N$-body model. The large filled cyan box presents the radial velocity and right ascension of NGC 2419. The black squares are CSS RRab's with distances $d_{G}>40 \mathrm{kpc}$.

(A color version of this figure is available in the online journal.)

near $\alpha=190^{\circ}$ appear to be associated with the leading arm of the Sgr stream. However, for the other Gemini stream RRab's, the velocities, like the distances, are not explained by the Law \& Majewski (2010) $N$-body simulations. In this regard, Sesar et al. (2012) noted that the RRab velocity measurements they found suggested the RRab's belonged to two distinct groups. Additionally, sources associated with our group D of Figure 9 (occurring near $\alpha=150^{\circ}$ ) have radial velocities that appear to vary rapidly with right ascension. These velocities are also inconsistent with the Law \& Majewski (2010) model, suggesting that they do not belong to the Sgr leading arm. The association between these feature-D RRab's and the Gemini stream RRab's is unclear, although values are similar.

\subsection{Links to NGC 2419}

As noted by Newberg et al. (2003), the outer halo BHB candidates they discovered reside near the unusual globular cluster (GC) NGC 2419. This system is located at $\alpha=114.53$, $\delta=38.88$ and distance $d_{h}=82.6 \mathrm{kpc}$ (Harris 1996, 2010 edition). The corresponding coordinates in the Majewski et al. (2003) Sgr coordinate system are $\Lambda=201.7, B=-8.5$, making it well within the limits of the Sgr stream system (Koposov et al. 2012). The Galactocentric radial velocity is given by Newberg et al. (2003) as $-14 \mathrm{~km} \mathrm{~s}^{-1}$, and Baumgardt et al. (2009) find an internal velocity dispersion of $4 \mathrm{~km} \mathrm{~s}^{-1}$.

NGC 2419 is noted as being one of the most metal-poor GCs ([Fe/H] -2.1; Mucciarelli et al. 2012). The cluster is notably old, with age 12.3 Gyr according to Forbes \& Bridges (2010). The system has the highest luminosity $\left(M_{V} \sim-9.6 \mathrm{mag}\right)$ of any GC with a Galactocentric distance $R>15 \mathrm{kpc}$, apart from the likely Sgr-dwarf-associated GC, M54 (Cohen et al. 2010). The half-light radius of this cluster is $19 \mathrm{pc}$ making it significantly more extended in the $\log \left(R_{h}\right)$ versus $M_{V}$ plane than other GCs with $R>15 \mathrm{kpc}$ (Mackey \& van den Bergh 2005). Indeed, the exceptional nature of NGC 2419 relative to outer halo GCs led van den Bergh \& Mackey (2004) to suggest that the object is the stripped core of a former dwarf spheroidal galaxy (dSph). Based on abundance studies, Cohen et al. (2010) and Cohen \& Kirby (2012) also found that the NGC 2419 appears like no other globular cluster, but rather the core of an accreted dwarf galaxy.
From Figures 7 and 17 we see that the location and radial velocity of NGC 2419 are a relatively good match for the Gemini tidal stream. The average velocities and metallicities of the Sesar et al. (2012) Cancer group B $\left(\langle\mathrm{v}\rangle_{\mathrm{gsr}}=16.3 \pm 7.1 \mathrm{~km} \mathrm{~s}^{-1}\right)$ and $[\mathrm{Fe} / \mathrm{H}]=-2.1 \pm 0.4 \mathrm{dex})$ and the metallicity of the Cancer group $\mathrm{B}$ are in reasonable agreement with the values expected for a stream from NGC 2419. However, the velocities and metallicities do not provide a strong enough association to link these sources.

Another important point to consider in the possible association between the Gemini RRL and NGC 2419 is the proximity of the sources. Some of the RRL have $\delta<20^{\circ}$ near the right ascension of the NGC 2419 ( $\alpha=114.53)$. Thus, if one was to assume that the Gemini tidal stream proceeds in the direction of the Sgr stream (as suggested by SDSS HB candidates), the stream stars would have to be dispersed across the entire $\sim 20^{\circ}$ of the Sgr system between the MLS RRab's and NGC 2419. Sources within this gap are not covered by MLS observations because of the coverage limits of the survey. It thus remains unclear whether there is any link between the Gemini stream and NGC 2419. Also, as noted earlier, our data show that the most distant Gemini RRab's are OoI type stars while NGC 2419 is well known to be OoII type. Indeed, Mucciarelli et al. (2012) found that NGC 2419 exhibits very little spread in $[\mathrm{Fe} / \mathrm{H}](\sigma=0.11 \mathrm{dex})$ suggesting it could not be linked with metal-rich RRL in the Gemini structure.

\section{DISCUSSION AND CONCLUSIONS}

We have performed a periodicity analysis of 3.1 million variable star candidates selected from photometry taken by the MLS survey and uncovered 1207 RRL (of which 538 are new). Comparison of the periods for the $\sim 600$ previous known RRL shows that the sources are accurately measured. We have also discovered 2040 new RRab stars in a re-analysis of CSS photometry.

Using Catalina Surveys photometry we have determined the nature of $90 \%$ of the SEKBO (Keller et al. 2008) RRL candidates and find that $60 \%$ are likely to be RRL. Our analysis of the SEKBO RRL candidates revealed selection of a pure set of RRab's with accurate average magnitudes requires many observations. The importance of repeated observations for characterizing variable star types was also recently demonstrated by Sesar et al. (2010), who used RRab light curves to study overdensities that had earlier been attributed to RRL in Sesar et al. (2007). They discovered that a number of the over densities attributed to RRL, based on photometric selection coupled with a small number of observations, were in fact due to intrinsically fainter $\delta$ Scuti stars as well as non-variable sources. Overall they found that only $70 \%$ of their initial candidates were RRL stars. Similarly, Ivezic et al. (2005) found that although it was possible to completely color-select a small fraction $(6 \%)$ of RRL based on SDSS photometry, if one wanted to select $60 \%$, a $72 \%$ non-RRL contamination rate would result. Similar levels of contamination make it equally difficult to trace halo structures using photometrically selected BHB stars.

In our analysis we also found a significant group of RRL with average $V$ magnitudes $\sim 20.5$. By combining these sources with CSS RRab's and photometrically selected HB candidates, we find strong evidence for a tidally disrupted stellar stream crossing $>60^{\circ}$ of the sky at Galactocentric distances from 70 to $110 \mathrm{kpc}$. This result confirms the existence of a stream first noted by Newberg et al. (2003). Comparison with halo density models shows that the feature is significant and that the halo 
density declines rapidly beyond $30-50 \mathrm{kpc}$, as previously noted by Watkins et al. (2009) and Sesar et al. (2010). However, since these results, like those of Watkins et al. (2009) and Sesar et al. (2010), are based on a thin slice through the halo, caution has to be taken when interpreting the extent of density regions outside the observed fields.

Although the Gemini RRL overlap with the Sgr stream system, we find that the large distances are inconsistent with existing Sgr models. This result is in agreement with Ruhland et al. (2011) and a recent sample of RRL discovered by Sesar et al. (2012). Furthermore, we find that the radial velocities of the RRL are inconsistent with simulations of the Sgr stream. However, we note that models of the Sgr stream system as a whole remains poorly constrained by observations.

We have investigated the possible relationship between the Gemini tidal stream and NGC 2419 as first proposed by Newberg et al. (2003). The most recent analysis of NGC 2419 shows significant evidence for it being the nuclear remnant of a disrupted dwarf galaxy (van den Bergh \& Mackey 2004; Mackey \& van den Bergh 2005; Cohen et al. 2010, 2011; Forbes \& Bridges 2010; Cohen \& Kirby 2012). Although, we find that the distances of many of the RRLs and HB candidates are consistent with NGC 2419, the available velocities and location of the Gemini stream are not in sufficient agreement to link the two structures. Furthermore, the most distant of the RRab's discovered appear to be metal-rich sources and would thus be inconsistent with stars observed in NGC 2419. Nevertheless, given the location of NGC 2419 within the halo, and within $10 \mathrm{deg}$ of the Sgr stream plane, it seems possible that a stream associated with NGC 2419 could join it to the Sgr system. This may in part account for the significant diversity in metallicity observed for varying Sgr stellar streams (Law \& Majewski 2010). Furthermore, as the photometric-selected HB candidates and RRab's cover the Sgr stream system and exhibit a distance gradient, it is possible that there is a second galaxy remnant associated with the Sgr stream. Such a source might explain the origin of the two intersecting streams of the Sgr system that has now been well delineated with MSTO stars by Belokurov et al. (2006) and Koposov et al. (2012). However, even with $40 \%$ uncertainties in the distances to MSTO stars (Newby et al. 2011), the Gemini stream RRab's are twice as distant as expected for MSTO stream stars (Koposov et al. 2012). Alternately, the Gemini stream may originate from the remnant of another disrupted dwarf galaxy that lies beyond the Gemini stream stars and is yet to be discovered. The Gemini stream leads into the Galactic plane beyond $100 \mathrm{kpc}$. A highly extincted system in the Galactic plane would be very difficult to detect.

Future photometric and spectroscopic observations of the $\mathrm{HB}$ stars within $10^{\circ}$ of NGC 2419 could confirm whether there truly is a tidal stream of RRL associated with NGC 2419. For example, the seemingly unique $\mathrm{Mg}$ and $\mathrm{K}$ abundance patterns in NGC 2419 found by Mucciarelli et al. (2012) would chemically tag stars originating from this source, even in the presence of overlapping tidal streams. If the Gemini tidal stream does follow the path expected from the SDSS HB candidates, additional deep photometric observations undertaken by projects such as LSST (Abell et al. 2009) should reveal numerous additional RRL along this tidal stream. Moreover, if these stars are associated with NGC 2419, we predict that the RRL will mostly be type Oosterhoff-II.

CRTS and CSDR1 are supported by the U.S. National Science Foundation under grants AST-0909182 and
CNS-0540369. The CSS survey is funded by the National Aeronautics and Space Administration under Grant No. NNG05GF22G issued through the Science Mission Directorate Near-Earth Objects Observations Program. J.L.P. acknowledges support from NASA through Hubble Fellowship Grant HF-51261.01-A awarded by the STScI, which is operated by AURA, Inc., for NASA, under contract NAS 5-26555. Support for M.C. and G.T. is provided by the Ministry for the Economy, Development, and Tourism's Programa Inicativa Científica Milenio through grant P07-021-F, awarded to The Milky Way Millennium Nucleus; by Proyecto Basal PFB06/2007; by Proyecto FONDECYT Regular 1110326; and by Proyecto Anillo ACT-86. SDSS-III is managed by the Astrophysical Research Consortium for the Participating Institutions of the SDSS-III Collaboration. Funding for SDSS-III has been provided by the Alfred P. Sloan Foundation, the Participating Institutions, the National Science Foundation, and the U.S. Department of Energy Office of Science. The SDSS-III Web site is http://www.sdss3.org/.

\section{REFERENCES}

Abell, P. A., Allison, J., Anderson, S. F., et al. (LSST Science Collaborations and LSST Project) 2009, LSST Science Book, Version 2.0, arXiv:0912.0201 Adelman-McCarthy, J. K., Agueros, M. A., Allam, S. S., et al. 2007, ApJS, 172, 634

Akhter, S., Da Costa, G. S., Keller, S. C., \& Schmidt, B. P. 2012, ApJ, 756, 23 Baumgardt, H., Cote, P., Hilker, M., et al. 2009, MNRAS, 396, 20

Bell, E. F., Xue, X. X., Rix, H., Ruhland, C., \& Hogg, D. W. 2010, AJ, 140, 1850

Belokurov, V., Evans, N. W., Bell, E. F., et al. 2007, ApJL, 657, L89

Belokurov, V., Zucker, D. B., Evans, N. W., et al. 2006, ApJL, 642, L137

Bertin, E., \& Arnouts, S. 1996, A\&AS, 117, 393

Blazhko, S. 1907, AN, 175, 325

Bono, G., Caputo, F., \& Stellingwerf, R. F. 1995, ApJS, 99, 263

Brown, W. R., Geller, M. J., Kenyon, S. J., et al. 2005, AJ, 130, 1097

Cacciari, C., Clementini, G., Prevot, L., et al. 1987, A\&AS, 69, 135

Catelan, M. 2009, Ap\&SS, 320, 261

Catelan, M., \& Cortés, C. 2008, ApJL, 676, L135

Clube, S. V. M., Evans, D. S., \& Jones, D. H. P. 1969, MmRAS, 72, 101

Cohen, J. G., Huang, W., \& Kirby, E. N. 2011, ApJ, 740, 60

Cohen, J. G., \& Kirby, E. N. 2012, ApJ, 760, 86

Cohen, J. G., Kirby, E. N., Simon, J. D., \& Geha, M. 2010, ApJ, 725, 288

Djorgovski, S. G., Drake, A. J., Mahabal, A. A., et al. 2011, in The First Year of MAXI: Monitoring Variable X-Ray Sources, ed. T. Mihara \& N. Kawai (Tokyo: JAXA Special Publ.), 263

Drake, A. J., Catelan, M., Djorgovski, S. G., et al. 2013, ApJ, 763, 32 (DR13) Drake, A. J., Djorgovski, S. G., Mahabal, A. A., et al. 2009, ApJ, 696, 870

Eggen, O. J., Lynden-Bell, D., \& Sandage, A. R. 1962, ApJ, 136, 748

Forbes, D. A., \& Bridges, T. 2010, MNRAS, 404, 1203

Freeman, K., \& Bland-Hawthorn, J. 2002, ARA\&A, 40, 487

Grillmair, C. J. 2006, ApJL, 645, L37

Harris, W. E. 1996, AJ, 112, 1487 (2010 edition)

Ibata, R. A., Gilmore, G., \& Irwin, M. J. 1994, Natur, 370, 194

Ibata, R. A., Lewis, G. F., Irwin, M., Totten, E., \& Quinn, T. 2001, ApJ, 551,294

Ivezic, Z., Lupton, R., Schlegel, D., et al. 2004, in ASP Conf. Ser. 327, Reaching to the Edge of the Milky Way Halo with SDSS, ed. F. Prada, D. Martinez Delgado, \& T. J. Mahoney (San Francisco, CA: ASP), 179

Ivezic, Z., Smith, J. A., Gajus, M., et al. 2007, AJ, 134, 973

Ivezic, Z., Vivas, A. K., Lupton, R. H., \& Zinn, R. 2005, AJ, 129, 1096

Jones, R. V., Carney, B. W., \& Latham, D. W. 1988, ApJ, 326, 312

Juric, M., Ivezic, Z., Brooks, A., et al. 2008, ApJ, 673, 864

Kato, T., Imada, A., Uemura, M., et al. 2009, PASJ, 61, 395

Keller, S. C., Murphy, S., Prior, S., Da Costa, G., \& Schmidt, B. 2008, ApJ, 678,851

Kinemuchi, K., Smith, H. A., Wozniak, P. R., et al. 2006, AJ, 132, 1202

Kollmeier, J. A., Gould, A., Shectman, S., et al. 2009, AJ, 705, L158

Koposov, S. E., Belokurov, V., Evans, N. W., et al. 2012, ApJ, 750, 80

Larson, S., Beshore, E., Hill, R., et al. 2003, BAAS, 35, 982

Law, D. R., \& Majewski, S. R. 2010, ApJ, 714, 229

Layden, A. C. 1997, PASP, 109, 524 
Liu, T., \& Janes, K. A. 1989, ApJS, 69, 593

Lomb, N. R. 1976, Ap\&SS, 39, 447

Mackey, A. D., \& van den Bergh, S. 2005, MNRAS, 360, 631

Majewski, S. R., Skrutskie, M. F., Weinberg, M. D., \& Ostheimer, J. C. 2003, ApJ, 599, 1082

Miceli, A., Stubbs, C. W., Hawley, K. H., et al. 2008, ApJ, 678, 865

Mucciarelli, A., Bellazzini, M., Ibata, R., et al. 2012, MNRAS, 426, 2889

Nemec, J. M. 2004, AJ, 127, 2185

Newberg, H. J., Yanny, B., Cole, N., et al. 2007, ApJ, 668, 221

Newberg, H. J., Yanny, B., Connie, R., et al. 2002, ApJ, 569, 245

Newberg, H. J., Yanny, B., Grebel, E. K., et al. 2003, ApJL, 596, L191

Newberg, H. J., Yanny, B., \& Willett, B. A. 2009, ApJL, 700, L61

Newby, M., Newberg, H. J., Simones, J., Cole, N., \& Monaco, M. 2011, ApJ, 743,187

Pietrukowicz, P., Udalski, A., Natal, D. M., et al. 2012, ApJ, 750, 169

Prior, S. L., Da Costa, G. S., \& Keller, S. C. 2009a, ApJ, 704, 1327

Prior, S. L., Da Costa, G. S., Keller, S. C., \& Murphy, S. J. 2009b, ApJ, 691,306

Rebassa-Mansergas, A., Gansicke, B. T., Schreiber, M. R., Koester, D., \& Rodriguez-Gil, P. 2010, MNRAS, 402, 620

Ruhland, C., Bell, E. F., Rix, H-W., \& Xue, X-X. 2011, ApJ, 731, 119

Scargle, J. D. 1982, ApJ, 263, 835

Schlegel, D. J., Finkbeiner, D. P., \& Davis, M. 1998, ApJ, 500, 525

Schwarzenberg-Czerny, A. 1989, MNRAS, 241, 153
Searle, L., \& Zinn, R. 1978, ApJ, 225, 357

Sesar, B. 2012, AJ, 144, 114

Sesar, B., Cohen, J. G., Levitan, D., et al. 2012, ApJ, 755, 134

Sesar, B., Ivezic, Z., Grammer, S. H., et al. 2010, ApJ, 708, 717

Sesar, B., Ivezic, Z., Lupton, R. H., et al. 2007, AJ, 134, 2236

Shapiro, S. S., \& Wilk, M. B. 1965, Biometrika, 52, 591

Sirko, E., Goodman, J., Knapp, G., et al. 2004, AJ, 127, 899

Smith, H. A., Catelan, M., \& Kuehn, C. 2011, in Carnegie Observatories Series, Vol. 5, RR Lyrae Stars, Metal-Poor Stars, and the Galaxy, ed. A. McWilliam (Pasadena, CA: The Observatories of the Carnegie Institution of Washington), 17

Soszyński, A., Udalski, A., Szymański, M. K., et al. 2009, AcA, 59, 1

Stepien, K. 1972, AcA, 22, 175

van den Bergh, S., \& Mackey, A. D. 2004, MNRAS, 354, 713

Vivas, A. K., \& Zinn, R. 2006, AJ, 132, 714

Vivas, A. K., \& Zinn, R. 2008, AJ, 136, 1645

Vivas, A. K., Zinn, R., Andrews, P., et al. 2001, ApJL, 554, L33

Watkins, L. L., Evans, N. W., Belokurov, V., et al. 2009, MNRAS, 398, 1757

Watson, C., Henden, A. A., \& Price, A. 2006, SASS, 25, 47

Welch, D. L., \& Stetson, P. B. 1993, AJ, 105, 1813

Yanny, B., Constance, R., Newberg, H. J., et al. 2009, AJ, 137, 4377

Yanny, B., Newberg, H. J., Kent, S., et al. 2000, ApJ, 540, 825

Zinn, R., \& West, M. J. 1984, ApJS, 55, 45

Zorotovic, M., Catelan, M., Smith, H. A., et al. 2010, AJ, 139, 357 